\title{
Stability of trajectories from Poincaré to Birkhoff: approaching a qualitative definition
}

\author{
Tatiana Roque
}

Received: 14 July 2010 / Published online: 9 April 2011

(C) The Author(s) 2011. This article is published with open access at Springerlink.com

\begin{abstract}
The article investigates the different conceptions of stability found in qualitative studies on the solutions of differential equations. We start from the definitions proposed by Poincaré and criticized by Birkhoff for not being fully qualitative, and show that the clarification of the criterion for stating that a property is qualitative comes precisely with Birkhoff. In addition, we note that the stability conceptions of Lyapunov and Levi-Civita are also important in this transition from the appearance of the first qualitative tools in the study of differential equations to the definition of stability in use in dynamical systems theory. The history of stability can help to explain the meaning of the word "qualitative" in this context.
\end{abstract}

\section{Introduction}

At the end of the nineteenth century, Henri Poincaré proposed a qualitative approach for the analysis of the solutions of differential equations. His article "Sur les courbes définies par une équation différentielle"1 is well known as a starting point in this kind of treatment (Poincaré 1881, 1882, 1885a, 1886). Its third and fourth parts, published, respectively, in 1885 and 1886, introduce some conceptual tools that would later prove useful for his research on celestial mechanics, and in the 1890s he wrote two further works on this same topic, (Poincaré 1890) and (Poincaré 1892-1899). As we will see later in this article, one of the main features of a qualitative treatment is to regard

1 "Sur les courbes" from now onwards in this article.

Communicated by : Jeremy Gray.

T. Roque $(\bowtie)$

Instituto de Matemática, Universidade Federal do Rio de Janeiro, P.O. Box 68530,

Rio de Janeiro, RJ 21941-909, Brazil

e-mail: tati@im.ufrj.br 
differential equation solutions as trajectories of a moving body. In this context, the term "trajectory" designates a solution.

Today, the qualitative analysis of differential equations is defined as the direct study of the geometry or topology of the set of solutions. This treatment has replaced the search for the properties of individual solution given by means of series developments. However, this is merely the current mathematical interpretation. The different meanings that the word "qualitative" has assumed throughout history still need a proper investigation.

It is worth noticing that Poincaré did not oppose qualitative and quantitative approaches. One important historical question thus arises: when did mathematicians start to distinguish qualitative from quantitative methods? It is our intention to answer the question in the particular context of stability. In our perspective, an inquiry into the development of the different definitions of stability emerges as an occasion to understand the exact meaning of the adjective "qualitative" when associated with the new methods introduced by Poincaré.

Several historians of mathematics have already studied the works of Poincaré on the qualitative theory of differential equations, but not with the same concerns as ours. ${ }^{2}$ One of the first works on the topic was written by Christian Gilain in 1977. In Gilain (1977), he analyzes the paper "Sur les courbes" in detail, showing its relationship with traditional research works on differential equations. Gilain (1991), relates the methods that Poincaré uses to describe the global aspect of two dimensional trajectories to the theory of algebraic curves and some aspects of his treatment of solutions are compared with those of other mathematicians. Jeremy Gray (1992) offers a wide account of how Poincaré uses the mathematics of his time, especially topology, in his qualitative works, and explains their application to different topics such as planetary astronomy, dynamics, and celestial mechanics.

Jean-Luc Chabert and Amy-Dahan Dalmedico (1992) study in the new methods introduced by Poincaré in "Sur les courbes" and in his works on celestial mechanics, highlighting their geometrical nature. In a historical research more concerned with the heritage of Poincaré, Dahan-Dalmedico (1996) summarizes his most important qualitative results, focusing on their reception by different mathematicians in different countries. Special attention is given to Birkhoff, considered "the only true disciple of Henri Poincaré in the West" (Dahan-Dalmedico 1996, p. 24). Poincaré's articles on the three body problem are discussed in depth in the book of Barrow-Green Poincaré and the Three Body Problem (Barrow-Green 1997). In Barrow-Green (1994), she had already analyzed the erroneous paper that Poincaré presented to the competition that celebrated the 60th birthday of King Oscar of Sweden and Norway in 1889 (Poincaré 1889). The efforts made to correct the error gave rise to another paper published in 1890 (Poincaré 1890). Barrow-Green compares both versions explaining the context in which they appeared.

All these works mention the question of stability, but their goal is not to proceed into a deep investigation of the different mathematical ways in which this concept can be defined. More recently, in a PhD thesis about the conception of "general" in

\footnotetext{
2 Nabonnand (2000) gives an account of the historical studies about Poincaré in various domains, and in particular in the qualitative theory of differential equations.
} 
the works of Poincaré, Robadey (2006) notes that there are two different notions of stability in the two versions of the paper submitted to the competition. We will return later to her remarks on the subject in order to stress the different notions of stability found in the qualitative works of Poincare and some of his followers. Other research works related to stability problems, treated from a qualitative point of view, will also be studied, such as the work of Aleksandr Mikhailovich Lyapunov.

Mawhin (1994) discusses the novelty of Lyapunov's works on differential equations and the theory of stability, paying special attention to the new fields he opened in mathematics and in applied domains (such as control theory). He also makes a comparison between Lyapunov and Poincaré and comments on the reception of their works by the French research community on differential equations. This last topic is analyzed in detail in (Mawhin 1996). In the context of Italian mathematics, we will look at Tullio Levi-Civita's works on stability and qualitative analysis, which have already been studied in Dell'Aglio and Israel $(1989,1987)$. These authors highlight the innovative character of Levi-Civita's approach to the three body problem, but also what he owes to tradition.

In all these historical texts, the definitions of stability proposed by each of the mathematicians appear as part of a whole analysis of their works. Here, we aim to draw on all these references, returning to the primary sources whenever necessary, but focusing on the mathematical definitions of stability. Our question is not to analyze the work of a particular mathematician. Rather, we seek the relations between the different stability definitions proposed by each mathematician, trying to identify to which cultural context the definition of stability belongs.

This article thus differs from the works cited above as it does not aim to study a particular mathematician, stressing the originality of his work. We propose a history of the mathematical definitions that the concept of stability has been associated with, presenting the different meanings it has assumed at the hands of different mathematicians, which were all recognized as belonging to the same field. Nor do we wish to analyze a particular problem concerning stability, such as the problems of the stability of the solar system or the stability of equilibria. They give rise to different mathematical definitions of stability, and our goal is only to inquire into the links between them, instead of describing stability results in a specific problem. ${ }^{3}$

Why this special interest on the question of stability? The topic has particular relevance for the constitution of the new mathematical discipline now called dynamical systems theory. As often occurs in the history of mathematics, a new field gains an identity when some procedures already in use are isolated and discussed. The first mathematician to propose a reflection on the nature of qualitative methods and the novelty they represent was George David Birkhoff. These questions emerged in his works in the 1910 s in connection with a discussion of the most adequate stability definition to be used in a qualitative treatment.

\footnotetext{
3 A brief description of some stability conceptions found in the works of Lagrange, Poincaré, and Lyapunov, and related to dynamical systems and modern control theory, is given by Leine (2010). Notwithstanding the legitimate goal of showing the differences between the definitions put forward by each mathematician, the article is not concerned with historical analysis.
} 
The expression "dynamical systems"-in the context of mathematical studies on differential equations - appears for the first time in the title of one of his articles "Quelques théorèmes sur le mouvement des systèmes dynamiques", presented in 1909 to the American Mathematical Society and published in 1912 (Birkhoff 1912). He defined a dynamical system as any system of ordinary differential equations in which the independent variable is interpreted as being the time, while other variables are coordinates of a "movement". Birkhoff (1927a) published the first treatise on the domain called Dynamical Systems, aiming to present to a large public the main definitions and conceptual tools that distinguish this new discipline from the traditional field of research devoted to differential equations.

We do not deny that Poincaré was the true creator of the qualitative approach, since he proposed key methods for a new treatment of differential equations. However, in order to identify the birth of a new theory, it is necessary to go beyond the search for methods now recognized as pertaining to this theory. Indeed, we can say that the field of dynamical systems was not created until its methods were explicitly defined as qualitative as opposed to the older ones. From this standpoint, the theory as such started with the works of Birkhoff, in particular those in which he discussed the appropriate qualitative definition for stability. A criterion for distinguishing different definitions of stability and their role in the characterization of qualitative methods became clear in Birkhoff's works from the 1920s on. Eventually, he (Birkhoff 1935, p. 310) explicitly affirmed that the term "stability", once applied to the property of Poisson periodicity (defined below) that had been taken into consideration by Poincaré 50 years before, was "unfortunate".

Therefore, we have a twofold goal. First, we want to show that the works of Poincaré do not represent a break with the classical research in differential equations and celestial mechanics. His writings on these topics combine, in an indifferent manner, traditional and original methods proposed by him for the first time. The task of the historian is not only to identify what is new in his works, but also what they take from the previous tradition. Our second goal is a consequence of the first, as we seek to show that a criterion for distinguishing a qualitative approach appears previously in the work of Levi-Civita, and then in Birkhoff's, in connection with the discussion on the many ways stability can be defined.

This distinguishes our case study of stability from other historical studies on qualitative methods. By elucidating the many different concepts of stability, particularly in the work of Birkhoff, it became possible for dynamical systems to emerge as a mathematical theory-more precisely as a mathematical theory of a qualitative nature.

Our choice of the texts to be analyzed follows Birkhoff's suggestions. He mentioned several texts of Poincaré and questioned his definitions of stability. Some works by Levi-Civita, in particular an article of 1901, were mentioned by Birkhoff as having provided a more adequate stability conception for a qualitative treatment. This mathematician made reference to Lyapunov's work on the stability of movements, although most of his texts had not yet been translated at that time.

We start in the following section by examining the traditional conceptions of stability in use in the treatment of solar system and problems concerning the stability of equilibria during the second half of the nineteenth century, which directly influenced 
Poincaré and Lyapunov. We close the section with a list of the stability definitions to be analyzed.

The third section describes the context in which Poincaré defined stability in the article "Sur les courbes" and shows how this definition is used in his works on celestial mechanics. Stability appears there in association with a special kind of periodicity for an individual trajectory, connected with its long term behavior. Nonetheless, there is an implicit conception of stability throughout his works, which is revealed when he requires the trajectories to be confined within some region in their domain.

We consider this conception to be implicit because the mathematical field of dynamical systems did not exist at that time. From the moment the qualitative theory was isolated as a specific approach, we claim that a stability conception proper to this domain had already been present in the works of Poincaré, but only in retrospect. We will explore the different definitions of stability in those works. An important example is the stability of a periodic solution, which will play a central role in our analysis.

The notion of stability used in the three body problem is very different from the one employed in problems involving the stability of equilibria, even though both are formulated by the same mathematicians. The question in the stability of equilibria is to investigate what happens when the initial conditions are perturbed, and therefore it engages in an analysis of the entire set of trajectories. A definition of the same kind for an arbitrary trajectory has been called "perturbative" stability by Birkhoff.

In the fourth section, we consider the differences between stability definitions used for an individual trajectory and the ones proposed directly for the whole set of trajectories. In this context, Lyapunov introduced a new mathematical characterization of stability. Such step is fundamental inasmuch as it allows a clarification of the meaning of "qualitative" when it appears associated with a mathematical definition of stability.

An analogous definition was employed by Levi-Civita in his works on the three body problem. By making an innovative use of the methods proposed by Poincaré, he applied the stability of equilibria to the study of a neighborhood of a periodic trajectory. Our fifth section is devoted to his synthesis of the two contexts in which stability was developed: the stability of equilibria and the three body problem.

Finally, in the sixth section, we explain what Birkhoff calls "qualitative stability". He identified different types of stability. One of them, formal stability, reveals the remains of a strong influence of analytical methods; another one is perturbative by nature and can adequately be called "qualitative". In the particular case of periodic solutions, Birkhoff showed that the situation named "stable" by Poincaré is in fact unstable, when a more appropriate stability definition is employed.

The new field of dynamical systems became autonomous in relation to the classical domain of differential equations with Birkhoff. Our conclusion briefly analyzes how this domain is inseparably linked to a general study of the different possible types of trajectories that are solutions of general systems. The problem of stability in a qualitative approach becomes a sub-problem of this more global one. 


\section{Stability problems and the traditional methods to treat them}

Physical-mathematical problems concerning stability were very common in the second half of the nineteenth century and covered a wide range of fields: elasticity, principles of mechanics, engineering mechanics, hydrodynamics, potential theory, thermodynamics, economic equilibrium, etc. ${ }^{4}$ It is not our intention to analyze stability conceptions in all these domains. We are only interested in the stability definitions of celestial mechanics, in particular those related to two problems: stability of the solar system and stability of the equilibrium figures of rotating fluids.

\subsection{Stability of the solar system}

Efforts to study the stability of the solar system, or more precisely the stability in the three body problem, motivated the development of specific analytical methods for the solution of differential equations. In the eighteenth century, Euler, Clairaut, and D'Alembert had already applied series developments of the solutions in order to study the perturbations in Keplerian movements of two bodies caused by the attraction of a third body. Lagrange and Laplace gave a sophisticated mathematical treatment to the problem, studying the variations of elliptical orbit elements under the effect of those perturbations (Lagrange 1774, 1781-1782; Laplace 1796, 1891-1904).

Lagrange started from the fact that the motion of a planet remains close to a Keplerian ellipse over a short duration. He could then use the ellipse as the basis for a coordinate system and write the differential equations that determine the variations of orbital elements in the elliptic motion, under the effect of perturbations caused by other planets, in order to show that they should be confined within fixed limits. These variations may be periodic - this implies that the planetary system will return to the neighborhood of its initial situation — or secular. In this case, there are long-term variations in the planets' semi-major axes that can accumulate with increasing time, thus producing great changes in the original aspect of the orbits. The traditional treatment of stability tried to eliminate the secular terms in order to demonstrate that the variation in elements of the planets' orbits would be confined within well-determined limits. Therefore, complete stability, in the sense of Lagrange and Laplace, was equivalent to the confinement of the variations of the orbital elements within certain limits.

If we consider first order terms in the masses and first degree terms of eccentricities and inclinations, the orbits of the planets are described by means of a linear differential system with constant coefficients. It was necessary to solve a characteristic equation and to find its roots (now called the "eigenvalues" of the matrix that defines the system). The numerical determination of these values was not an easy task, as there was a considerable number of variables. But stability was defined by means of some conditions that these values under consideration took. For instance, the existence of two

\footnotetext{
4 For a broad panoramic description of research works on stability in different countries in this period see Chap. 14 of (Grattan-Guinness 1997) named An era of stability: mechanics, 1860-1900. Stability criteria in hydrodynamics are deeply studied in (Darrigol 2002).
} 
equal roots indicated the possibility of a collision between two planets, thus preventing a system from being stable. ${ }^{5}$

The calculations made by Lagrange and Laplace showed that, up to first order in the planets' masses, the secular variations vanish. On the other hand, considering not only linear terms but also the squares and cubes of the masses in the series development, Poisson (1808) showed that the series could contain terms in which the sine is multiplied by time, implying that the amplitudes of variations of the semi-major axis can grow indefinitely with time. So, the system returns to the neighborhood of its initial configuration but can also go far away from it. Stability had thus a new meaning according to Poisson. While for Lagrange, the approximate solutions must be bounded in the neighborhood of the elliptical orbits in order to guarantee stability, for Poisson, the solutions can go far away from the periodic orbit, but at some time they return to its neighborhood. Poisson stability conception implies a special kind of periodicity for solutions that we will call, from now on, "Poisson periodicity". 6

The two conceptions of stability - as confinement and as Poisson periodicity-also appear in the works of Poincaré. Before that, in the middle of the nineteenth century, the astronomer Le Verrier went back to the calculations of Lagrange and Laplace and considered the effects of higher order terms in the series. Le Verrier (1856) showed that these terms led to significant alterations in the orbits, and that the previous calculations could not be used for an indefinite amount of time. The astronomer improved the precision of the former methods by considering non-linear terms in the series developments that depend on a parameter (such as mass, eccentricity, or inclination).

Laskar (1992) explains Le Verrier's methods in detail and shows how he found that other phenomena than non-periodic secular terms can also harm stability. Second (or higher) order terms in the solutions may have large coefficients that render them more important than first order terms. This phenomenon, known as the problem of "small divisors", is caused by very small quantities appearing in the denominator of some terms in the solution. The complications due to these small divisors revealed that the convergence of the series depends on initial conditions. In Le Verrier's treatment, if the first terms of the series representing an element of the orbit decrease rapidly enough, one can infer stability. So, for him, the question of stability had to do with some kind of astronomical convergence.

From the second half of the nineteenth century to the beginning of the twentieth century, there was a proliferation of essays trying to improve Le Verrier's approach. Astronomers such as Newcomb, Lindstedt, and Gyldén proposed new methods that took into account higher order terms and prevented time from appearing outside trigonometric terms. A history of these methods is still to be done, but it is out of our scope in this paper to analyze these works in detail. A detailed description of perturbation

\footnotetext{
5 The methods developed to demonstrate stability included not just techniques of series development, but also algebraic methods that did their way far beyond celestial mechanics. As Brechenmacher (2007) shows, Lagrange's method to determine these characteristic values was an algebraic practice used by a network of mathematicians in the period.

6 We use this expression to prevent associating the conception with the mathematical definition it acquires later as "Poisson stability".
} 
methods in celestial mechanics in this period can be found in (Tisserand 1889-1896). ${ }^{7}$ For our purposes, the only germane point is the common approach according to which the secular terms should be excluded from the series development. This implied an identification of the criterion of stability with the reduction of series development to strictly trigonometric terms.

The main mathematical tools used in the astronomical works mentioned above are perturbation methods. They consist of mathematical methods that start from the exact solution of a related problem in order to find an approximate solution to a problem that cannot be solved exactly. In our case, the three body problem is described by means of a perturbation of an elliptical movement.

The simplified form of the problem is a Keplerian ellipse, which is correct when there are only two gravitating bodies (as, for e.g., the Earth and the Moon). The resolved but simplified problem is then perturbed by considering the gravitational attraction of a third body (the Sun). The conditions met by the corrected solution do not match the equation required by reality (the law of attraction, in the example), but the correction aims to provide a better approximate answer.

If one of the masses is considered to be much smaller than the other two, the three body problem can be formulated by adding a "small" term to the mathematical description of the two-body problem. The procedure leads to an expression of the desired solution in terms of a power series (or trigonometric series) in some "small" parameter that quantifies the deviation from the solvable problem. The leading term in this power series is the solution of the solvable problem whereas further terms describe the deviation in the solution due to another deviation from the initial problem. Formally speaking, the full solution approximation $X$ is given by a series in a small parameter (here called $\epsilon$ ):

$$
X=X_{0}+\epsilon X_{1}+\epsilon^{2} X_{2}+\ldots
$$

In this example, $X_{0}$ is the known solution of the Keplerian problem and $X_{1}, X_{2}$, ... are higher order terms that may be found iteratively by means of some systematic procedure. If for small $\epsilon$ these terms become successively smaller, the approximate solution of the non-exact problem does not differ too much from the exact solution of the solvable problem (periodic solution). The approximate solution may differ considerably from the periodic solution at some instant of time, but then returns infinitely often to its neighborhood. In short, if we analyze the behavior of higher order terms in the series development of a solution and conclude that this implies confinement or Poisson periodicity of the approximate solution, we say that this solution is stable.

\subsection{Stability of equilibria}

For our purposes in this article, we can say that the question of the stability of equilibria starts with Lagrange, as he was the first one to formulate precise criteria to verify stability in a mechanical system. In the Méchanique Analytique (Lagrange 1788), he

\footnotetext{
7 More recent references are cited in Taton et al. (2009).
} 
affirmed that an equilibrium position is stable if the system, assumed in equilibrium and displaced by a small amount, tends to return to this position by itself. The most prominent result of Lagrange's treatment consisted of his statement that the equilibrium is stable if the potential function is in a minimum when the system is in equilibrium.

From a mathematical point of view, there is an important difference between the definition of the stability of equilibria and the one employed to study the stability of the solar system. In the latter case, the perturbation methods give an approximate solution of a perturbed system, and we would like to investigate the long-term stability of this individual solution. In the first case, as in the problems treated in this section, the stability is a property of a solution (equilibrium point) that is verified by an analysis of the behavior of other solutions in its neighborhood. These solutions are obtained by a perturbation of the initial conditions.

Using a power series approximation of the potential function up to the second order, Lagrange showed that the equilibrium is stable when first-order terms vanish and second-order terms are positive, which corresponds to a minimum of the potential function. Lejeune-Dirichlet (1846) improved the proof by showing that higher order terms might also correspond to a minimum of the potential function (Lagrange's earlier proof based upon linearization was not sufficient). The stability result is now known as "Lagrange-Dirichlet theorem".

Concerning the stability of equilibrium figures of a rotating mass of fluid in the nineteenth century, we should mention the works of several mathematicians, such as Jacobi, Liouville and Riemann, as discussed in Lützen (1984). However, in the period we are analyzing here, the second half of the nineteenth century, the most influential reference, for stability of equilibrium, is Thomson and Tait's treatise, published in 1867. In its second edition, which appeared between 1879 and 1883 (Thomson and Tait 1879-1883), they added a more complete discussion of the stability of equilibrium figures of a rotating fluid. The authors analyzed the stability of Jacobi and Maclaurin ellipsoids using a criterion based on the energy function.

As Norton Wise points out, Thomson and Tait's book is "the first major work in any language to transform Newtonian and Lagrangian mechanics into the new dynamics of energy in the second half of the 19th century" (Wise 2005, p. 521). Motion stability, more particularly that of equilibrium, was one of their main concerns:

There is scarcely any question in dynamics more important for Natural Philosophy than the stability and the instability of motion. We therefore (...) propose to give some general explanations and leading principles regarding it (Thomson and Tait 1879-1883, Sect.346, p. 416).

Thomson and Tait's intuitive definition of the stability of equilibria proposed just afterwards was analogous to Lagrange's. If a material system, under the influence of internal and applied forces, and varying according to some definite law, is displaced infinitely little in any direction from a particular position of equilibrium, and left to itself; and if then it commences and continues to vibrate, without ever experiencing more than an infinitely small deviation in any of its parts from the position of equilibrium, then the equilibrium in this position is said to be stable. But this definition does not indicate how to verify stability, and a mathematical criterion was then enunciated. 
A mass of fluid is in a stable equilibrium if the potential energy function has a minimum in this state. Similarly, when viscosity is disregarded, the equilibrium is stable if the kinetic energy attains a maximum. This criterion was largely known before Thomson and Tait, but their treatise made this method popular. Of particular importance was the redefinition of the criterion in the context of Hamiltonian dynamics.

Although Hamilton had reformulated mechanics long before, his approach was not taken up in mathematical physics until it appeared in Thomson and Tait's book, as Wise (2005, p. 528) remarks. The equations of motion were derived from the energy principle, written in variational form. This implied several theorems about minimum and maximum properties of the kinetic energy of mechanical systems. Thus, as their approach to the problem was based on Hamiltonian dynamics, the principle of stability outlined by Lagrange was developed in this new context. The behavior of the system was determined by means of the roots of the characteristic equation. If its roots are all real and negative, the equilibrium is stable.

A remarkable point to observe in Thomson and Tait's treatise is the introduction of considerations on the stability of general motions, not necessarily in equilibrium. In Section 347 of Thomson and Tait (1879-1883), they asserted that an actual motion of the system is said to be stable if every possible infinitely small conservative disturbance of this motion gives rise to an alteration bringing the system again near the undisturbed path. This must also happen in a finite time and without more than an infinitely small digression. If these conditions are not fulfilled, the motion is said to be unstable.

Soon afterwards, in Sect. 356, Thomson and Tait assumed the need for a mathematical criterion for stability, which must be based on the principle of varying action. The problem of investigating the effect of an infinitely small disturbance, produced at any instant in the motion of any conservative system, may then be reduced to a practical problem of mathematical analysis.

In fact, in the following years, the definition of stability and the mathematical condition founded on properties of the kinetic energy were mixed up. A stable equilibrium then started to be defined as an extremum point of the energy function. The intuitive definition based on perturbed and unperturbed motions could not be transformed into a mathematical definition as these notions (of motions, perturbed and unperturbed) were not mathematically defined. While the space of motions was not defined as a mathematical object, the condition for stability served as its definition. ${ }^{8}$

Poincaré and Lyapunov had declared that their own works on the stability of equilibria departed from Thomson and Tait's treatment, by aiming to go beyond their unjustified use of linear approximations. But while Poincaré used the same definition based on an energy function, as we will later see Lyapunov proposed a new definition, implying a direct consideration of the space of motions. He thus proceeded to give a stability definition that is independent from the mathematical condition involving properties of the energy function. The question of qualitative stability has to do with

\footnotetext{
8 This question has to do with the history of the conception of "phase space", or "space of configurations". It is not our goal here to trace the history of this notion, but if it were the case, we would have to mention the work of Liouville that probably inspired Poincaré and Lyapunov, see Lützen (1984).
} 
a definition that can be stated directly in the space of motions, and does not involve analytical expressions for integrals or approximate solutions.

\subsection{Sketch of Poincaré and Lyapunov stability definitions}

In order to make the distinctions clear before placing them in their historical context, we list below the definitions of stability to be considered later. In the works of Poincaré, stability considerations appear from 1885, when the problem of solving a differential system of equations was restated to bring out its intimate connection with the problems of celestial mechanics (Poincaré 1885a). As an example, from this moment onwards the solutions of the system were called "trajectories". The stability of a trajectory in three dimensions was defined as a kind of periodicity.

Stability as Poisson periodicity (Poincaré): Let $M$ be the point of the trajectory corresponding to an instant $t$. If we trace a circle of radius $r$ around $M$, this trajectory is stable if the points in the trajectory of $M$ enter this circle infinitely many times, even if $r$ is made arbitrarily small.

This property was later called "Poisson stability", as the system can go far away from the initial state but it returns to its neighborhood in the future. This is the explicit definition stated by Poincare in the paper "Sur les courbes". But there is another implicit conception of stability, as we will see in the third section, implying that the trajectories remain confined in a limited region of space.

In his works on celestial mechanics, these two conceptions appear again, and a third definition was introduced. Periodic solutions become central and Poincaré defined their stability by reducing the analysis of the solutions of a system of differential equations in the neighborhood of a periodic solution $x_{i}=\varphi_{i}(t)$ to the study of the linear system:

$$
\frac{\mathrm{d} \xi_{i}}{\mathrm{~d} t}=\sum_{j=1, n} \frac{\partial X_{i}}{\partial x_{j}} \xi_{j}
$$

where $\varphi_{i}(t)+\xi_{i}(t)$ are perturbations of the periodic solution. The numbers $\alpha_{i}$ in

$$
\xi_{1}=e^{\alpha_{1} t} S_{1, k}, \ldots, \xi_{n}=e^{\alpha_{n} t} S_{n, k}
$$

are called "characteristic exponents" ( $S_{i, k}$ are periodic solutions with the same period of $\varphi_{i}(t)$ that can be written as absolutely and uniformly convergent trigonometric series).

Stability of periodic solutions (Poincaré): The periodic solution $\varphi_{i}(t)$ is stable if all characteristic exponents $\alpha_{i}=a_{i}+b_{i} i$ are purely imaginary numbers. This works as a guarantee that all $\xi_{i}$ remain finite, since $\xi_{i}=\left(\cos \left(b_{i} t\right)+i \sin \left(b_{i} t\right)\right) S_{i, k}$, where $S_{i, k}$ are periodic functions.

We observe that the stability has to do with the possibility of expressing the solutions by means of trigonometric functions, as in the classical treatment of celestial mechanics. However, this definition is not for an individual trajectory. The stability 
of a periodic solution takes into consideration the behavior of other solutions in its neighborhood.

Whereas Poincaré presented the first definition of stability in the third part of "Sur les courbes", in the same year of 1885 , he also published an important paper on the stability of rotating figures of a fluid. The stability definition in there follows the traditional form used by Lagrange, Thomson, and Tait.

Stability of equilibrium (Poincaré): Let the position of a system be defined by $n$ quantities $x_{1}, x_{2}, \ldots, x_{n}$ and assume the existence of a force function $F\left(x_{1}, x_{2}, \ldots, x_{n}\right)$. We have an equilibrium position when the derivatives of this function vanish and this equilibrium is stable when this function has a maximum.

Lyapunov considered the domain of application of this approach quite limited, as it is only valid when an integral (the force function) is given. It was thus necessary to develop methods that enable this problem to be solved without integrating. He defined stability in another manner that takes the behavior of perturbed and non perturbed motions into consideration.

Stability of equilibrium (Lyapunov): Let $\left(x_{1}, \ldots, x_{n}\right)=(0, \ldots, 0)$ be an equilibrium solution of a differential system

$$
\left\{\begin{array}{l}
\frac{\mathrm{d} x_{1}}{\mathrm{~d} t}=X_{1}(x, t) \\
\cdots \\
\frac{\mathrm{d} x_{n}}{\mathrm{~d} t}=X_{n}(x, t)
\end{array}\right.
$$

This solution is stable if, for each positive number $l$, arbitrarily small, there is another positive number $\epsilon$ such that $\left\|x_{1}(t)\right\|<l, \ldots,\left\|x_{n}(t)\right\|<l$ for all positive values of $t$, provided that $\left\|x_{1}\left(t_{0}\right)\right\|<\epsilon, \ldots,\left\|x_{n}\left(t_{0}\right)\right\|<\epsilon$.

So, if the initial conditions of a trajectory are near the equilibrium state, the trajectory will always remain within a small neighborhood of this point. The stability of a general solution $\left(f_{1}(t), f_{2}(t), \ldots, f_{n}(t)\right)$ is analyzed by departing from the above definition. The transformation

$$
\left(x_{1}, x_{2}, \ldots, x_{n}\right) \rightarrow\left(f_{1}(t), f_{2}(t), \ldots, f_{n}(t)\right)+\left(x_{1}, x_{2}, \ldots, x_{n}\right)
$$

is used in order to reduce the question about the stability of the general solution to the stability of the equilibrium solution as defined above for a differential system that fulfils

$$
\left(X_{1}(0, t), X_{2}(0, t), \ldots, X_{n}(0, t)\right)=(0,0, \ldots, 0)
$$

Intuitively speaking, a general solution is stable if the solutions obtained by an $\epsilon$-perturbation of its initial condition remain close.

Both definitions proposed by Poincare in the context of the three body problem-that of stability as Poisson periodicity of an individual trajectory and the one of a periodic trajectory stability - were criticized by Birkhoff. In the article "Quelques théorèmes sur le mouvement des systèmes dynamiques" (Birkhoff 1912), he introduced special solutions called "recurrent movements", which are useful for the 
description of the whole set of trajectories. This definition ends up by calling into question, a few years later, the first definition of stability proposed by Poincaré. The property of Poisson periodicity should be used, according to Birkhoff, to characterize a recurrent movement, while stability should be defined in terms of other criteria.

The way Poincaré defined stability of a periodic solution was also questioned. In Birkhoff's view, a definition that demands a series development to contain only trigonometric terms must be considered as a kind of "formal" or "astronomical" stability. Still employing Birkhoff's designations, the true "qualitative" stability is the one defined directly on the set of trajectories, which establishes stability for a solution in terms of the behavior of other solutions in its neighborhood. He called a definition of this kind "perturbative" (as opposed to formal), or "mathematical" (as opposed to astronomical) stability.

What Birkhoff called "perturbative" stability has essentially the same nature as the definition put forward by Lyapunov. ${ }^{9}$ Yet it has been used in the study of stability in the three body problem. Levi-Civita defined stability of a periodic trajectory by means of the same criterion of Lyapunov's equilibrium stability. In his turn, Birkhoff departed from the works of Levi-Civita to show that it is necessary to go beyond Poincaré's definition.

It is important to stress the fact that the word "perturbative" is employed here with a different meaning from the one it used to have in the so-called "perturbative methods of celestial mechanics". There, the conception of stability is formal, as the criterion applied lies in the possibility of writing the solution as a specific series. As we have already explained, perturbative methods of celestial mechanics deal with the actual problem as a perturbation of an exact solvable problem and attempt to investigate the stability of a solution of the perturbed problem in terms of the properties of its series expansion. The notion of perturbation is not employed to define stability. In Lyapunov's definition, the stability of a solution is defined in terms of the behavior of a perturbed solution of the same differential problem. As Birkhoff finally made explicit, the perturbative character of the stability criterion is what renders its definition properly qualitative.

\section{The context of Poincaré stability definitions}

\subsection{The qualitative methods of Poincaré}

In his paper "Sur les courbes", Poincaré started with a study of the solutions of a real differential equation of the first order and first degree with polynomial coefficients:

$$
\frac{\mathrm{d} x}{X}=\frac{\mathrm{d} y}{Y}
$$

where $X$ and $Y$ are polynomial functions in $x$ and $y$.

\footnotetext{
9 Birkhoff did not quote Lyapunov as the first to propose this definition, but we will see later that his works were indirectly influenced by the Russian's through the intermediation of Levi-Civita.
} 
In the third part, published in 1885 , this same equation was written considering the independent variable as time:

$$
\frac{\mathrm{d} x}{\mathrm{~d} t}=X, \quad \frac{\mathrm{d} y}{\mathrm{~d} t}=Y
$$

and $x$ and $y$ as the coordinates of a moving point. From this moment on, the solutions of the system received the name of "trajectories", which expresses an intimate relationship with the problems of celestial mechanics. After this reformulation, the set of solutions could be conceived as a family of trajectories of a mobile point with the variable time implicitly taken into consideration.

In the first two parts of the memoir "Sur les courbes", the problem of stability was already considered, in relation with the description of the global aspect of trajectories in two dimensions. The results obtained for two dimensional solutions are highlighted as being of a topological nature by Gray (1992). By means of the classification theorem ${ }^{10}$ we can conclude that, either a trajectory is closed (stable), or it tends asymptotically to a singularity without ever getting back near to its initial state (unstable).

Poincaré shows it is true by means of the definition of what he calls "cycles without contact". These are closed curves that are not solutions of the equation, but curves that solutions can cross only once. Hence, if a trajectory crosses a cycle without contact it cannot return to a neighborhood of the initial point and we can say it is unstable. The verification of instability is easier than that of stability, since it is possible to test, by means of the cycles without contact, if a solution definitely leaves a region of the domain. Stability arises when a solution remains indefinitely in a region of the domain, what is much more complicated to establish.

In order to investigate the neighborhood of periodic trajectories in three dimensions, Poincare introduced, in the fourth part of "Sur les courbes", what we call the section method. ${ }^{11}$ We explain here how this procedure allowed him to reduce the problem to two dimensions.

One supposes that $s$ is a closed curve representing a periodic solution and one draws a plane normal to the trajectory that intercepts it at a point $P$. A system of coordinates can be defined (Poincaré 1886, p. 199) in this plane, that is called "plane of section". The periodic solution is represented by a fixed point $P$, and if $P_{0}$ is a point in the normal plane, the trajectory that passes through $P_{0}$ will intersect the normal plane once again at a point $P_{1}$ (Fig. 1). ${ }^{12}$

Poincaré studied the transformation ( $T$ in the figure) that takes $P_{0}(=x$ in the figure) to $P_{1}(=T(x)$ in the figure). He showed that this transformation is holomorphic, and then he analyzed its development in series.

\footnotetext{
10 Nowadays known as "Poincaré-Bendixon theorem", see also Gilain (1991). In the case of the solar system, the problems need larger dimensions.

11 Poincaré himself had given no special name to this method.

12 Poincaré supposed the continuity of solutions with respect to the initial conditions.
} 
Fig. 1 Transformation $T$ (defined on the section plane) taking $x$ to $T(x)$

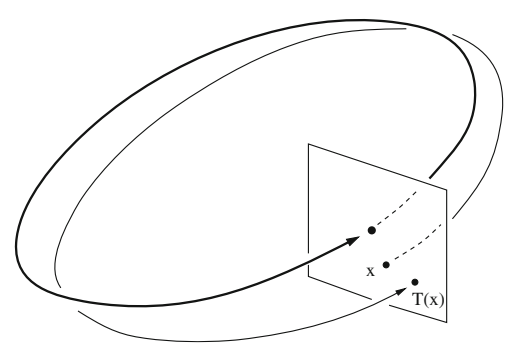

Fig. 2 The four types of singularity
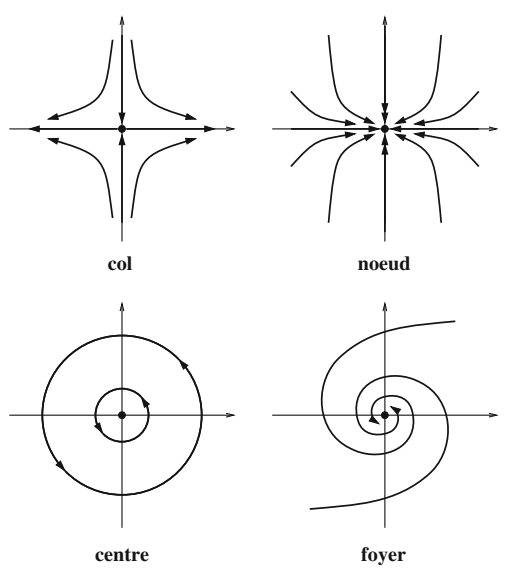

The terms of first order have the form:

$$
\left\{\begin{array}{l}
x=A_{1} e^{\lambda_{1} t} \varphi_{1}(t)+A_{2} e^{\lambda_{2} t} \varphi_{2}(t) \\
y=A_{1} e^{\lambda_{1} t} \psi_{1}(t)+A_{2} e^{\lambda_{2} t} \psi_{2}(t)
\end{array}\right.
$$

where $A_{i}$ are constants and the functions $\varphi$ and $\psi$ are trigonometric series. The $\lambda_{i}$ are given by the equation $S^{2}-(\alpha+\delta) S+(\alpha \delta-\beta \gamma)=0$, with $S=e^{2 \lambda \varpi}$. The possible situations were then examined using the values $\lambda_{1}$ and $\lambda_{2}$.

If these values are real and positive, one of them greater and the other less than one, then Poincaré demonstrated that, in the plane of section, there are two invariant curves $K$ and $K^{\prime}$, which means that if a point $P_{0}$ is on one of the curves, the next intersection $P_{1}$ will also be on the same curve. Such a conclusion, later generalized by Hadamard, Lattès and Birkhoff, was extremely important for later research works. ${ }^{13}$

It is possible to study what happens in the neighborhood of the point $P$ using the same methods that were employed to analyze the neighborhood of the singularities in

\footnotetext{
13 Hadamard (1901) extended Poincaré's outgoing results to non-analytic cases. Lattès (1906) showed that the characteristic exponents are essential elements for the problem, and that the study of invariant curves is possible and useful for more generic cases than those examined by Poincaré. The exact definition of surface of section and that of invariant curves was given in (Birkhoff 1920b). For a history of the section method and the consequences of its application to the definition of a dynamical system, see Roque (2007).
} 
Fig. 3 Surface without contact (dotted line) with a closed curve as intersection with the section plane

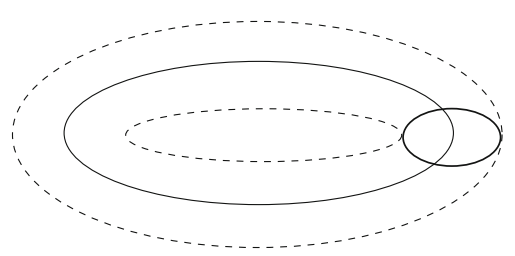

two dimensions. These singularities had been classified in terms of nodes (noeuds), centers (centres), focuses (foyers) and saddle-points (cols) (Fig. 2). ${ }^{14}$

The intersection points in the plane of section behave in a manner analogous to these four possible neighborhoods of singularities in two dimensions. ${ }^{15}$

Instead of cycles without contact there will now be surfaces without contact. If we have, in the plane of section, the case analogous to the center singularity in two dimensions, in which the fixed point is surrounded by closed trajectories, we can build up surfaces without contact around the periodic solution, which reduce to cycles without contact in the plane of section. If there is a surface without contact in its neighborhood, the periodic trajectory is unstable, because a trajectory that passes through a neighboring initial condition leaves and cannot return. However, a conclusion in favor of stability is much more difficult to obtain (Fig. 3).

Poincaré proposed to investigate the question through the existence of an invariant integral. ${ }^{16}$ This is a real positive function defined in local coordinates inserted in the neighborhood of the closed trajectory that preserves volume. It means that, if a set of points in the neighborhood of the closed trajectory have a certain volume at time $t$, the images of those points by the function will be a set having this same volume for all subsequent instants of time. If this function exists, there is no surface without contact. This is a first step towards stability. But in this case, there still may be instability. The reason for that lies in the presence of small divisors, which can make the series expressing the solutions diverge. Because of that, it is easier to infer instability. The presence of a cycle or a surface without contact implies instability, but the absence of this feature does not imply any positive result about stability. ${ }^{17}$

At the very end of "Sur les courbes", Poincaré analyzed the neighborhood of a periodic trajectory and concluded that a stability result is hard to obtain whatever definition is used for stability. The only explicit definition was that of Poisson periodicity, but he provided the following example, where different conceptions about the mathematical definition are put together.

\footnotetext{
14 See Gilain (1991) and Gray (1992).

15 This fact really impressed Poincaré, and it is at the origin of the study of two-dimensional point transformations, see also Roque (2007).

16 This name was given later in Poincaré (1890), where he showed that the existence of an invariant integral is a fundamental property of Hamiltonian systems. As Barrow-Green points out (1997, p. 40), it was only in this article, where the idea of an invariant integral played an essential role in stability arguments, that Poincaré developed a coherent theory about invariant integrals.

17 Poincaré (1890) showed that the integral curves are contained in level surfaces over which energy remains constant. Hence, the trajectories do not go from one level surface to another, which means that there are no surfaces without contact able to be crossed by integral curves. Even though it does not guarantees stability.
} 
Poincaré studied the particular case of a three-dimensional system having a periodic solution, written in coordinates $(\omega, \phi, \rho)$. The surfaces $\rho=$ constant are mutually enveloped tori (nested one into the other), defined around the periodic solution. The behavior of a variable $\rho$ allows the stability of the solution to be studied, since it measures the distance between the points of the analyzed trajectory and the closed trajectory. Several cases can happen (Poincaré 1886, p. 221):

(1) Trajectories recede constantly or they approach the closed trajectory asymptotically. What may happen is that certain trajectories recede while others approach. Thus, Poincaré concluded that there is always instability, in the sense that a point close to the closed trajectory for $t=0$ will no longer be a neighbor for large positive values of $t$ (or it was not a neighbor for large negative values of $t$ ).

(2) A point close to the closed trajectory for $t=0$ remains constantly close for all positive and negative values of $t$, i.e., stability is guaranteed. In this case, $\rho$ oscillates constantly within certain limits passing an infinite number of times through all values between these limits.

(3) A point close to the closed trajectory for $t=0$ can recede far for certain values of $t$, and therefore the periodic solution is not stable. But, from another viewpoint this solution is stable since this point returns and becomes infinitely close to the closed trajectory for certain values of $t$. This happens whenever $\rho$ passes an infinite number of times through all possible values.

In the first case, Poincaré used the definition of stability as Poisson periodicity in order to conclude that there is instability. Nonetheless, in the second case, there is stability in a stronger sense than that of Poisson periodicity, as the other trajectories are confined in the neighborhood of the closed trajectory. It is precisely in this last sense that one can conclude in favor of instability in the third case. After all, the trajectories do not remain confined in a neighborhood of the periodic solution.

Different notions of stability coexist in the analysis of one single example. In addition to the issue of Poisson periodicity, what is at stake is the confinement of trajectories within some region in space, in this case, the neighborhood of a periodic solution.

\subsection{Stability as Poisson periodicity and stability as confinement}

In the third part of "Sur les courbes" Poincaré explicitly defined the stability of a three-dimensional trajectory of a differential system of first order and second degree:

It happens then that the trajectory cannot be a closed curve; but, nevertheless, it keeps a certain stability: one can even say that it is a periodicity of particular nature. In fact, let $M$ be a point in the trajectory that the moving point occupies in an instant $t$. We trace a circle around the point $M$ with an arbitrarily small radius $r$. The moving point starting at $M$ will obviously go beyond the circle, but it will cross again this small circle an infinite number of times, no matter how small $r$ can be (Poincaré 1885a, p. 92) [Poincaré's italics]. ${ }^{18}$

\footnotetext{
18 Il arrivera alors que la trajectoire ne sera pas une courbe fermée; mais néanmoins elle jouira d'une
} certaine stabilité: on peut même dire d'une certaine périodicité d'une nature particulière. En effet, soit $M$ 
Here, we note that stability is equivalent to the Poisson periodicity of an individual solution. A periodic trajectory goes an infinite number of times through all its states. A trajectory is stable if, $M$ being an arbitrary point, the trajectory returns an infinite number of times to an arbitrarily small neighborhood of this point. This type of stability was called in (Poincaré 1890) "Poisson stability". But this is not the only possible definition for the stability of a trajectories. All over the paper "Sur les courbes" the above definition goes together with different, however implicit, conceptions of stability.

In an article about the new ideas of Poincaré, Chabert and Dahan-Dalmedico (1992, p. 296) observe that stability as explicitly defined in "Sur les courbes" is an intrinsic property of a trajectory; and they stress the fact that, in his works on celestial mechanics, Poincaré defined stability on the set of trajectories. Nevertheless, still in "Sur les courbes", on the paragraph immediately after the definition mentioned above, Poincaré acknowledged that:

Stability defined in this fashion has solely a theoretical importance. In practical terms, it would be necessary to determine a region of space in which the moving point remains invariably confined (Poincaré 1885a, p. 94). ${ }^{19}$

Stability is now identified with the confinement of trajectories to a certain region of space. This property already implies an investigation of the whole set of trajectories in order to find regions in which trajectories must confined. This double conception of stability as Poisson periodicity and as confinement appeared again in Poincaré's papers on the three body problem and celestial mechanics. One particular fact to observe is the different emphasis on the type of stability required in the two versions of the article presented to the 1889 competition (Poincaré 1889).

Poincaré considered a system of ordinary differential equations:

$$
\frac{\mathrm{d} x_{i}}{\mathrm{~d} t}=X_{i}(i=1, \ldots, n)
$$

where $X_{i}$ are analytic functions of $x_{i}, \ldots, x_{n}$. As in "Sur les courbes", the $x_{i}$ are geometrically represented as the coordinates of a point $P$ in space that describe a trajectory when $t$ varies. In the very beginning of "Sur le problème des trois corps et les équations de la dynamique" in its first version (Poincaré 1889), the one which contained the mistake, he said there is stability when $x_{i}$ remains under certain limits as $t$ varies. This means that the trajectory of $P$ is confined within a limited region in space.

The set of trajectories that pass through a given curve in space is called a surface trajectory, and it cannot be cut by any other trajectory. Closed surface trajectories play an analogous role to the surfaces without contact and they provide the main tool

Footnote 18 continued

un point de la trajectoire, occupé en un temps $t$ par le point mobile. Décrivons autour du point $M$ un cercle de rayon $r$ aussi petit que nous voudrons. Le point mobile partant du point $M$ sortira évidemment de ce cercle, mais il viendra traverser de nouveau ce petit cercle une infinité de fois, et cela, quelque petit que soit $r$.

19 La stabilité ainsi définie n'a qu'une importance théorique. Pour la pratique, il faudrait déterminer une région de l'espace où le point mobile reste constamment renfermé. 
for verifying stability, as they divide the space into an interior and an exterior region. If the initial position of $P$ is in an interior region then the trajectory of this point is eternally confined in the same region, and there is stability.

In the first version, the main approach used by Poincaré to demonstrate stability was to search for closed surface trajectories. The clarity of this criterion contributes to the sense of optimism about the ultimate resolution of the stability problem, as noted by Barrow-Green, in opposition to the tenor of the second version (Barrow-Green 1997, p. 75).

As Robadey (2006, pp. 124-125) points out in, we can explain the different emphasis on stability in the two versions of the paper by comparing their introductions. In the first, the final goal, highlighted from the beginning, is to demonstrate stability by means of a combination of traditional and new geometrical methods. The definition of stability is almost always taken as the one mentioned above, seeking to confine trajectories in some region of space. Poisson periodicity is also cited as a possible conception of stability, but it remains isolated in a section containing some results about the almost periodic behavior of trajectories. ${ }^{20}$

Robadey also notes that, in the letters Poincaré exchanged with Mittag-Leffler (Poincaré and Mittag-Leffler 1999), it becomes clear that the competition jury criticized the stability definition used in the first version. In particular, Weierstrass contested the physical sense of this definition, as it does not consider the possibility of collisions within the region of confinement. In his response, Poincaré reaffirmed the first definition by showing that it excludes collisions. The Poisson periodicity conception had a secondary place up to this moment.

After the discovery of the error in the stability demonstration, this question lost importance and in the second version (Poincare 1890), the term "stability" appears much later than in the first. On the other hand, since, the Poisson periodicity of trajectories is announced in the introduction as a quite important result to be presented. In the restricted problem of three bodies, the initial conditions for $P$ may be chosen to ensure that the trajectory of this point returns an infinite number of times arbitrarily near its initial position. ${ }^{21}$ The term "stability" appears for the first time in the section devoted to this result, and not in the introduction.

As Barrow-Green shows, negative results acquire a more prominent place in the second version. The above conclusion thus is one of the few positive results to remain and to sustain hope for a positive response to the stability problem. In a letter sent to Mittag-Leffler on the 9th of December 1889, Poincaré affirmed that "stability subsists" in the sense above (Poincaré and Mittag-Leffler 1999, Letter 93). ${ }^{22}$ Poincaré (1890, p. 313) started to call "Poisson stability" the property that we have called up to now "Poisson periodicity". The theme of Poisson stability acquires even more importance in (Poincaré 1892-1899), where Poincaré includes an entire chapter on the subject.

\footnotetext{
20 Robadey calls "recurrence" what we have chosen to call "Poisson periodicity". In our view, the term "recurrence" is more mathematically charged. We prefer thus to name this property using another designation until the moment it gains a mathematical definition as "Poisson stability".

21 The proof of this result relies in Poincaré's "recurrence theorem", as explained in Barrow-Green (1997, p. 86).

22 Anne Robadey also cites this letter in Robadey (2006, p. 131).
} 


\subsection{Periodic solutions and their stability}

The definition of the stability of a periodic solution was made explicit by Poincaré in his works on the three body problem and celestial mechanics, (Poincaré 1890) and (Poincaré 1892-1899), even though it had already appeared in "Sur les courbes".

These works treat a special version of the three body problem in which there are two bodies of negligible mass, ${ }^{23}$ each moving according to a Keplerian ellipse around a third mass. In this case, there is an infinite number of periodic solutions. Poincaré asked when and how it is possible to use existing information about the behavior of this system to analyze the situation in which one of the masses - first considered zero-is considered infinitely small. In the third volume of Poincare 1892-1899, this problem received the name of "restricted three body problem".

It is a perturbation problem that ultimately depends on a parameter $\mu$ - the infinitely small mass mentioned in the previous paragraph — taken as zero in the Keplerian situation. Poincaré studied the perturbation effects of $\mu$ on the periodic solutions through the analytic continuation of one of these solutions for small values of $\mu .^{24}$ A large part of Poincaré's work on celestial mechanics is concerned with the question of (i) showing that periodic solutions exist and (ii) analyzing their behavior after small perturbations of parameter $\mu$.

The perturbation of this parameter produces another differential system. The question of stability of periodic solutions has to do with the behavior of other solutions for the same system, so it is a different question. To investigate a periodic solution stability is the first step for the study of other solutions (of the same system) in its neighborhood. ${ }^{25}$ If the initial conditions of a trajectory are slightly different from those corresponding to a periodic solution, what is its future behavior? Will the mere fact of having initial conditions close to the periodic solutions be enough to ensure a kind of "similar" behavior?

Before defining stability for periodic solutions, Poincaré introduced convenient coordinates enabling a study of the behavior of trajectories in their neighborhood. He wanted to describe solutions of a system of differential equations:

$$
\frac{\mathrm{d} x_{i}}{\mathrm{~d} t}=X_{i}\left(x_{1}, x_{2}, \ldots, x_{n}\right)
$$

in the neighborhood of a periodic solution $x_{i}=\varphi_{i}(t)$. Considering that the perturbations of $\varphi_{i}(t)+\xi_{i}(t)$ are also solutions, and discarding all non-linear terms, he wrote

\footnotetext{
23 For example, if the three bodies are the Sun, the Earth and the Moon, the last two masses can be neglected because they are very small when compared with the mass of the Sun.

24 Barrow-Green (1997, pp. 92-93) notes that the complete solution of the problem depends on precisions added after the second version of "Sur le problème des trois corps et les équations de la dynamique", when the use of Cauchy's majorants methods becomes more explicit.

25 Hill was the first to show the importance of periodic solutions in the three body problem in (Hill 1878). In his presentation of the moon theory (Gray 1992, p. 505), he used the periodic solutions as intermediate orbits for the study of trajectories in their neighborhood. For a detailed analysis of Hill's contribution to the solution of the Earth-Moon-Sun problem, see Gutzwiller (1998). Poincaré stated that it can be advantageous to consider a periodic solution as a first approximation of a solution. He then quoted Gyldén's works, and named it "intermediate orbit" (Poincaré 1892-1899, p. 81, vol. I).
} 
what he named the "equations of variation":

$$
\frac{\mathrm{d} \xi_{i}}{\mathrm{~d} t}=\sum_{j=1, n} \frac{\partial X_{i}}{\partial x_{j}} \xi_{j}
$$

Thus, Poincaré managed to reduce the initial problem of how to solve a non-linear differential equation in the neighborhood of a periodic solution to the problem of how to solve a homogeneous linear system in $\xi$, whose coefficients are periodic functions. There are $n$ numbers $\alpha_{i}$, called the "characteristic exponents" of the periodic solution, and a solution of the system can be written as a linear combination of $n$ solutions of the type:

$$
\xi_{1}=e^{\alpha_{1} t} S_{1, k}, \ldots, \xi_{n}=e^{\alpha_{n} t} S_{n, k}
$$

where the $S_{i, k}$ are periodic solutions with the same period of $\varphi_{i}(t)$ that can be written as absolutely and uniformly convergent trigonometric series. ${ }^{26}$

These characteristic exponents control the behavior of the linear system. By means of these values, Poincaré hoped to investigate the situations in which the perturbation of a periodic solution gives rise to another periodic solution, or to a solution not too distant from the periodic solution. This question is analogous to the one at the very end of the paper "Sur les courbes", studied in the example of the torus, but Poincarénow defined, in a formal manner, a periodic solution $\varphi_{i}(t)$ as stable if all characteristic exponents $\alpha_{i}=a_{i}+b_{i} i$ are pure imaginary numbers. This works as a guarantee that all $\xi_{i}$ remain finite since $\xi_{i}=\left(\cos \left(b_{i} t\right)+i \sin \left(b_{i} t\right)\right) S_{i, k}$, where $S_{i, k}$ are periodic functions. $^{27}$

The demand that the characteristic exponents are pure imaginary numbers assures that trajectories that are near to the periodic solution in some instant cannot recede too much. But such conclusion, only valid for the linear approximation provided by the equations of variation, is associated with the existence of integrals that can be written in terms of a combination of trigonometric series. This assures a kind of formal stability, in the later sense of Birkhoff.

We will be able to see in the next sections that the question is treated by LeviCivita and Birkhoff in a different manner from Poincaré's. Both showed that Poincaré's so-called "stable" case is in fact unstable, according to what they regard as a more adequate definition of stability. Levi-Civita observed that, if one considers non-linear perturbations, it is possible that a solution close to the periodic one leaves its neighborhood and never returns.

Indeed, Poincaré did not go far in his study of stable periodic solutions. Using geometric methods, which were to have a strong influence on Levi-Civita and Birkhoff,

\footnotetext{
26 The procedure is similar to the one previously used in Poincaré's section method. However, here the continuous three-dimensional system is reduced to another continuous two-dimensional linear system, while there we have discrete iterations of points on the transverse section.

27 The condition for the characteristic exponents $\alpha_{i}$ be pure imaginary can be expressed with a demand that the characteristic values $e^{\alpha_{i}}$ have module 1 (for they would then be over the trigonometric cycle). Both formulations were used.
} 
he studied the neighborhood of an unstable periodic solution. He expected to solve the problem of three bodies by showing that trajectories do not recede too much from a neighborhood of the periodic solution, i.e., they remain confined in a region of space.

Instability of a periodic solution could be established whenever one can find, in its neighborhood, other solutions receding from it. But stability was still associated with the existence of trigonometric series, as in the classical methods of celestial mechanics.

In the neighborhood of an unstable periodic solution there is a family of asymptotic solutions. These are curves that asymptotically tend to the periodic solution with increasing or decreasing time, and are represented geometrically by two asymptotic surfaces. ${ }^{28}$ Poincaré used the section method in order to study these surfaces. The intersection of the periodic solution with the section plane is a fixed point, and by the same procedure the asymptotic surfaces turn into curves over the plane. If these curves meet to form a closed curve, there is a set of asymptotic solutions that remains confined to a given region of space, and we can infer stability.

The mistake Poincare had made in the first version of the paper presented to the competition (Poincaré 1889) was exactly at this point: these asymptotic curves actually intersect, but do not form a closed curve as he had thought. These are the so called "doubly asymptotic" trajectories. ${ }^{29}$ Their existence implies that the behavior of trajectories in the neighborhood of the unstable periodic solution may be very complex: a doubly asymptotic trajectory can begin by being very close to the periodic solution when $t$ is large and negative; but then it moves away and deviates greatly from the periodic solution before getting close again to this solution when $t$ is large and positive. Moreover, the existence of a doubly asymptotic trajectory actually means that an infinite number of such trajectories exist. Levi-Civita and Birkhoff showed later that such complexity also appears in the neighborhood of a stable periodic solution, which forces a reassessment in the definition of stability.

\subsection{Main tools that will be used later in research work on stability}

We will resume in the next section to the analysis of Levi-Civita and Birkhoff notions of stability, focusing on their study of stability of periodic solutions. However, although their approach to this subject took them in a direction different from Poincaré's, the conclusions they obtained depend on several conceptual tools provided by him. We summarize the most important of them:

- the section method (used to analyze the neighborhood of a periodic solution)

- the invariant curves (which generalize the curves $K$ and $K^{\prime}$ discovered by Poincaré, invariant under the dynamics)

- the doubly asymptotic trajectories

- the geometric theorem on the existence of periodic solutions

\footnotetext{
28 Barrow-Green (1997, pp. 104-107) shows that the role of this geometric representation changes from the first to the second version of the paper on the three body problem.

29 These solutions were later called "homoclinic" in Poincare (1892-1899, V.III). In Anderson (1994), we find a brief description of Poincaré discovery of this type of trajectories.
} 
The three first tools have already been mentioned. The fourth one is the approach used in the "last geometric theorem" of Poincaré. In 1912, a few weeks before his death, Poincaré published the paper, (Poincaré 1912), where he enunciated a new theorem that, once demonstrated, would confirm the existence of an infinite number of periodic trajectories in the restricted three body problem for all values of parameter $\mu$.

He proposed new conceptual tools, taken from algebraic topology, in order to show that if a continuous, one-to-one, area-preserving transformation $T$ takes the ring $R$, formed by concentric circles of radii $a$ and $b(a>b)$, into itself in such a way as to move the points on the circle of radius $a$ in a positive sense and the points on the other circle in a negative sense; then there are at least two points of the ring that remain invariant under the iteration of $T$.

Barrow-Green (1997, pp. 169-173) analyzes the suggestions given by Poincaré and the final proof provided by Birkhoff in 1913. This result played a key role in the study of stability of periodic solutions, since the existence of points on the ring that remain invariant under the iteration of $T$ implies the existence of periodic solutions if the ring is thought of as being over the section (when we apply the section method).

In Sect. 6, we discuss some consequences of this result found by Birkhoff. But before analyzing how Levi-Civita and Birkhoff followed the works of Poincaré on the stability of periodic solutions, it is worthwhile to mention Lyapunov's previous stability definitions (even if they are not stated for periodic solutions).

\section{Stability directly defined on the set of trajectories}

Poisson stability is a property of an individual trajectory, while Poincaré's definition of stability for a periodic solution concerns the family of other solutions in its neighborhood. This last definition, provided in 1890, assures that the trajectories of neighboring points in a periodic trajectory do not go too far. However, this limited behavior is stated as a formal requirement regarding the existence of characteristic exponents.

Around the same year, Lyapunov also furnished a definition of stability for a trajectory in terms of the behavior of other trajectories in its neighborhood, but his criterion was directly given on the space of trajectories. A trajectory was defined as stable if a perturbation of the initial condition does not affect its behavior too much. Thus, before returning to the study of the three body problem, we can indulge in a sort of digression about some aspects of the works of Lyapunov, influenced by problems related with equilibrium stability.

\subsection{The exchanges between Poincaré and Lyapunov about stability of equilibrium}

As we have seen, the attempts made by Thomson and Tait to generalize the LagrangeDirichlet theorem for a fluid were quite popular at the time of Poincaré and Lyapunov. Nevertheless, they enunciated many results without proof and their demonstrations, when furnished, did not seem rigorous enough. Poincaré and Lyapunov took this gap 
as a point of departure for their researches, and shed new light over the problem of the stability of equilibria of a rotating fluid. ${ }^{30}$

Poincaré was interested in the different forms that figures of equilibrium can assume, and investigated bifurcation points of parameters related to these figures. He showed, for instance, that it is possible to obtain infinitely many equilibrium figures different from ellipsoidal ones, and the question of stability appears in this context. Lyapunov was instead more concerned with stability in its own, which means simply to inquire if the perturbed figures are close enough to the original one, no matter their form. Hence, even if their works have some points in common, they employ different definitions of stability.

One of Poincaré's most important articles on the equilibrium figures of a rotating fluid (Poincare 1885b) was published in the same year of the third part of "Sur les courbes". However, the notions of stability used in these works do not agree. It is interesting to observe that the qualitative methods Poincaré proposed in "Sur les courbes" were later elaborated by Lyapunov in his second thesis, of 1892, in order to deal with stability of equilibrium, something Poincaré did not do.

In fact, Lyapunov had already thought about the stability of equilibrium figures in 1884, during his first thesis, which was translated into French as "Sur la stabilité des figures ellipsoïdales d'équilibre d'un liquide animé d'un mouvement de rotation"(Lyapunov 1904). He used Thomson and Tait's stability principle in order to obtain a version of Lagrange's theorem for fluids, and hence to demonstrate the stability of ellipsoidal figures.

Soon after having concluded this work, Lyapunov heard of the papers of Poincaré on equilibrium figures of a fluid. This is the beginning of a correspondence that lasted two years (Smirnov and Youchkevitch 1987) and started when Lyapunov sent Poincaré a copy of his thesis. In Poincaré's answer, in October 1885, he suggested that Lyapunov read the paper he had just published in the Acta Mathematica, that is (Poincare 1885b).

Almost a year later, Lyapunov wrote again emphasizing that his own works deal mainly with the question of stability whereas Poincaré's are concerned with more general issues, such as the diversity of possible equilibrium figures. The main goal of Lyapunov was to demonstrate rigourously the result enunciated in the second edition of Thomson and Tait's treatise (Thomson and Tait 1879-1883), affirming that the equilibrium of a fluid is stable if the total energy has a minimum.

Having noticed a difference between his own definition of stability and the one Lyapunov proposed in his thesis, Poincaré asked for more clarification:

What should I understand by this new definition of stability which has to move away from the equilibrium figure after a sufficiently small perturbation of the fluid, and this at least as long as there will not come to the surface any projections under a form of nets or blades, no matter how thin one can suppose them; in other words as long as there will be no wrinkles. What are those projections,

\footnotetext{
30 For an introduction to the works of Poincaré and Lyapunov on the problem of equilibrium figures of a rotating fluid, see Gray (1992). For a comparative analysis of their works on differential equations see Mawhin (1994); we will only focus on the new definition of stability proposed by the Russian mathematician.
} 
those nets, those blades and those wrinkles? (Smirnov and Youchkevitch 1987, pp. 7-8). ${ }^{31}$

Lyapunov replied that his aim was to extract, from the energy equation, all the consequences related to stability, which requires a definition of the exact scope of the idea of two equilibrium figures being "infinitely close" to one another. If we consider generic deformations of the figure, it may be the case that, after infinitely small perturbations, the fluid figure has a distinct nature from the equilibrium figure, even if only because of the presence of very thin prominences. To exclude them it would be necessary to create hardly verifiable conditions on the nature of initial perturbations. Lyapunov stated accordingly:

As this is very hard, I have preferred to slightly modify the definition of stability, and the modification merely comes to the fact that one now must consider the equilibrium figure as being stable even in those cases when, after infinitely small perturbations, the figure of the liquid can move away from the equilibrium figure more and more if the spacing is made only by means of deformations which come together with infinitely close thin projections (Smirnov and Youchkevitch 1987, p. 9) [Lyapunov's italics]. ${ }^{32}$

The important aspect in this definition is that the stability criterion requires only that the figures to remain close to one another-they can have different natures. As we will see, the mathematical stability definition Lyapunov proposed in his second thesis had a clear influence on these considerations.

\subsection{Qualitative, or "perturbative”, stability}

The best known work of Lyapunov remains his second thesis, published in Russian in 1892 and translated into French 15 years later with the title Problème général de la stabilité du mouvement (Lyapunov 1907). ${ }^{33}$ In the foreword, Lyapunov mentioned Poincaré's paper "Sur les courbes" as having provided the methods to be generalized in a major set of applications. ${ }^{34}$

\footnotetext{
31 Que dois-je entendre par cette nouvelle définition de la stabilité qu'après une perturbation suffisamment petite le liquide doit s'écarter aussi peu qu'on voudra de la figure d'équilibre au moins aussi longtemps qu'il ne se produit pas, à la surface, de saillies sous forme de filets ou de lames, quelque minces qu'on les suppose, en d'autres termes pour qu'il n'y a pas de rides. Qu'est-ce que c'est que ces saillies, ces filets, ces lames et ces rides?.

32 Cela étant très difficile, j'avais préféré de modifier un peu la définition de la stabilité, et cette modification se réduit à ce qu'on doit regarder la figure d'équilibre comme stable même dans le cas quand, après les perturbations infiniment petites, la figure du liquide peut s'écarter de plus en plus de la figure d'équilibre, si cet écartement ne se fait que par le moyen des déformations qui s'accompagnent des saillies infiniment voisines minces.

33 An English translation of this book have been published in 1992 in a commemorative edition of its centenary (Lyapunov 1992).

34 At the end of the foreword Lyapunov comments on Poincaré's works on celestial mechanics, in particular the article "Sur les problème des trois corps et les équations de la dynamique", which came out when the book was already in print but that contains results analogous to his.
} 
In spite of the fact that Poincaré restricts his research to very special cases, he uses methods that allow much more general applications and they can still lead to several new results. We shall see that by what follows, for, mostly in my research, I have been guided by ideas developed in the already quoted paper (Lyapunov 1907). ${ }^{35}$

Like Poincaré, Lyapunov argued that it is necessary to develop a method capable of assuring stability in cases for which the integration of differential equations is not possible. The problem of the stability of equilibria consists in knowing if one can choose initial values small enough, so that, for all values of time after the initial instant, the solutions remain smaller than limits given in advance, no matter how small these limits are. If one can integrate the differential equations, or find a potential function, this problem is easy to solve. But Lyapunov emphasized that it would be important to develop methods that would allow one to verify the stability of a solution, independently of the possibility of integrating the differential system.

One of Lyapunov's main goals was to demonstrate the converse of the LagrangeDirichlet theorem. We know that a sufficient condition for stability is the potential function to have a maximum in the equilibrium position. But is this condition also necessary? He obtained this result in certain cases and provided procedures for treating equations with constant or periodic coefficients. The conceptual tools developed by Lyapunov also permit a study of stability in more general cases. The paper (Mawhin 2005) gives a good account of the most significant result in the book, we highlight here those aspects related to his concept of stability.

From the standpoint of a qualitative analysis, the most remarkable aspect put forward by Lyapunov is to have regarded the solutions as movements, and to have focused on properties related to the set of movements. This has enabled him to define some properties of solutions by means of a direct analysis of the set of movements. Lyapunov's second thesis begins by suggesting how a perturbed and a non-perturbed movement can be mathematically defined; what a perturbation is in itself; and how a perturbed movement can be defined as being near to a non-perturbed one. All these concepts had been used earlier, but mostly in an intuitive manner, without rigorous definitions. This new treatment can be explained by noting that Lyapunov's interests went beyond an analysis of stability by means of properties related to the potential function. It was thus necessary to define stability directly on the set of movements.

Lyapunov's definition of stability of equilibrium appears as follows: ${ }^{36}$

Let $\left(x_{1}, \ldots, x_{n}\right)=(0, \ldots, 0)$ be an equilibrium solution of a differential system

\footnotetext{
35 Bien que M. Poincaré se borne à des cas très particuliers, les méthodes dont il se sert permettent des applications beaucoup plus générales et peuvent encore conduire à beaucoup de nouveaux résultats. C'est ce qu'on verra par ce qui va suivre, car, dans une grande partie de mes recherches, je me suis guidé par les idées développées dans le Mémoire cité.

36 This definition is given in a different manner in the thesis introduction (Lyapunov 1907, pp. 209-210). There Lyapunov furnished a more general definition depending on the quantities (named $Q_{i}$ ) in relation to which stability is studied. But we find the exact definition given here in Lyapunov (1907, p. 466).
} 


$$
\frac{\mathrm{d} x_{1}}{\mathrm{~d} t}=X_{1}(x, t), \ldots, \frac{\mathrm{d} x_{n}}{\mathrm{~d} t}=X_{n}(x, t)
$$

This solution is "stable" if, for each positive number $l$, arbitrarily small, we can find another positive number $\epsilon$ such that whenever the functions $x_{i}$ have at initial instant real values satisfying $\left\|x_{1}\left(t_{0}\right)\right\|<\epsilon, \ldots,\left\|x_{n}\left(t_{0}\right)\right\|<\epsilon$, the inequalities $\left\|x_{1}(t)\right\|<$ $l, \ldots,\left\|x_{n}(t)\right\|<l$ are also satisfied for all positive values of $t$.

The stability of a general solution $\left(f_{1}(t), f_{2}(t), \ldots, f_{n}(t)\right)$ is defined by means of an equilibrium point stability. Lyapunov used the transformation:

$$
\left(x_{1}, x_{2}, \ldots, x_{n}\right) \rightarrow\left(f_{1}(t), f_{2}(t), \ldots, f_{n}(t)\right)+\left(x_{1}, x_{2}, \ldots, x_{n}\right)
$$

in order to reduce the question about stability of a general solution to the related question of the stability of equilibria as defined above.

The methods inspired by the paper "Sur les courbes" are used in order to detect stability in terms of regions in which the solutions, with initial conditions close to equilibrium, must be confined. Lyapunov employed his so-called second method to study stability in terms of the possibility of obtaining certain functions, defined in the neighborhood of equilibrium and not dependent on any explicit solution for the system. These functions, called "Lyapunov functions" nowadays, have to be found by trial and error methods. If a level surface of a Lyapunov function involves the equilibrium point, then a solution with initial condition close to that point cannot leave its a neighborhood of it, and the equilibrium is stable.

The role of these functions is somewhat analogous to those of cycles and surfaces without contact as proposed by Poincaré. The existence of a surface without contact characterizes instability. However, in Poincaré's works there were no criteria of the type that would ensure stability. Lyapunov's functions allow one not only to infer stability but also to discover the extension of the set of trajectories that do not recede from equilibrium, as well as the extension of the set of trajectories that tend to equilibrium. In a qualitative treatment of stability, which can be named "perturbative" after Birkhoff, given a movement, the question is precisely to analyze its relationship with other neighboring movements.

Jean Mawhin (2005, p. 672) notes that the methods developed by Lyapunov are more analytical than those of Poincaré. Despite this fact, his treatment of stability can be said to be more qualitative. There are two ways to study stability: find exact or approximate solutions of differential equations or obtain information about the set of its solutions without writing their analytical expressions. In Lyapunov's 1892 thesis, we find approaches, like the second method, that allow one to infer stability of solutions without any knowledge of their analytical expression.

Even if his definitions are less geometrical than Poincaré's, Lyapunov proposed new objects, like unperturbed and perturbed motions, that enable him to state properties directly in the space of motions. As a consequence, his definition of stability does not need to take into account the analytical expression of solutions. It can thus be said to be a qualitative definition, not because of the geometrical way in which it is stated, but as a definition given directly as a property of the space of motions. 


\subsection{Poincaré's and Lyapunov's conceptions of stability}

For our purpose in subsequent sections it is sufficient to retain the manner in which Lyapunov treated stability for an equilibrium point. If we remember Poincarés section method, the periodic solution reduces to a fixed point on the transverse section (the intersection of the periodic solution with the section). Since a fixed point is, with respect to a point transformation, analogous to an equilibrium point, the stability of a periodic solution can be conceived in the same way as the stability of equilibria.

Poincaré did not define the stability of a periodic solution in this way. In Poincaré 1890 and Poincaré 1892-1899, he did not employ the section method to define the stability of periodic solutions. He used the so-called "equations of variations" and tried to write the trigonometric functions that describe the variation of other solutions in a neighborhood of a periodic solution. As we have mentioned, in his works about celestial mechanics, Poincaré had used the section method to analyze what happens in the neighborhood of an unstable periodic solution.

We will see in the later sections, in a study more concerned with the stability of periodic solutions, that the definition proposed by Lyapunov is better for obtaining results on stability by means of a direct investigation of the geometric space of solutions. Therefore, it can be said to be more "qualitative" than Poincaré's definition of stability. ${ }^{37}$

Poisson stability can be seen as a definition that applies, or not, to a trajectory in the space of trajectories. But it concerns only an individual trajectory, and not the other ones in its neighborhood. As Barrow-Green (1997, p. 181) remarks, this stability definition given by Poincare is less restrictive than Lyapunov's. For a trajectory to be stable in Lyapunov's sense, all perturbed trajectories must remain close to it, and also each point in the perturbed motions has to be close to its contemporaneous point in the unperturbed motion. However, Lyapunov's definition of the stability of equilibria served as the prototype for the stability of a general motion. In the subsequent qualitative works concerning stability in the three body problem, in which the section method is employed to analyze the stability of periodic solutions, this definition was turned into the properly qualitative one.

In 1897, 10 years before the translation of his second thesis into French, Lyapunov presented a synopsis of some of the results achieved in his thesis in the article "Sur l'instabilité de l'équilibre dans certains cas où la fonction de forces n'est pas un maximum" (Lyapunov 1897), published in the Journal des Mathématiques. The main question was to demonstrate the converse of the known result about the stability of equilibrium: if the force function does not attain a maximum can we be sure that instability occurs?

This issue was responsible for the rare quotations of the author by his contemporaries, and we will summarize in the next part the relevant results cited by Levi-Civita.

\footnotetext{
37 Even in the general case, to analyze stability of an arbitrary trajectory, Lyapunov's definition has become more useful in qualitative approaches, as we can see in Cesari (1959) or La Salle and Lefschetz (1961).
} 


\section{Qualitative stability in the three body problem}

Levi-Civita was one of the first mathematicians to use the qualitative point of view introduced by Poincaré. We confine our inquiry to the long article titled "Sopra alcuni criteri di instabilitá" (Levi-Civita 1901). ${ }^{38}$ In this text, cited several times by Birkhoff, the author mentioned Lyapunov's first article in French (Lyapunov 1897), and regretted the fact that his work about the general stability of movement had only been published in Russian at that time. His acquaintance with Lyapunov's works does not go beyond the 1897 article and some synopses published four years before in the Jahrbuch über die Fortschritte der Mathematik.

Levi-Civita used the section method in order to reduce the problem of stability of a periodic solution to the related one concerning the stability of a fixed point over twodimensional section. He was the first to catch a glimpse of the possibility of defining stability for a periodic solution in the same manner as the stability of equilibria. In this sense, he produced a synthesis, using methods developed to study the stability of equilibria in the three body problem.

\subsection{Levi-Civita's synthesis}

The initial problem of (Levi-Civita 1901) is the same as that presented by Poincare in Les Méthodes nouvelles (Poincaré 1892-1899, V.III, Chap. XXVI). It begins with a "canonical" system of differential equations $\frac{\mathrm{d} x_{i}}{\mathrm{~d} t}=X_{i}$ ( (uch that $\sum \frac{\mathrm{d} X_{i}}{\mathrm{~d} x_{i}}=0$ ), possessing an integral invariant.

For a two-dimensional system $\frac{d x}{d t}=X, \frac{d y}{d t}=Y$, Levi-Civita noticed that Poincaré's research suggested that instability has a qualitative character - as it can be verified by means of inequalities - and stability is quantitative - as it requires conditions on the nature of functions $X$ and $Y$. We recall that Poincaré had already remarked that the use of cycles without contact to verify the fact of instability was precisely the mark that distinguished his own methods from traditional ones. In this sense, instability can be considered more qualitative than stability.

The verification of stability remained dependent upon formal conditions on the functions that intervene during the series development of $X$ and $Y$ - which, in certain cases, can be used to solve the problem by integration. An example of a quantitative condition on the nature of functions $X$ and $Y$ that permits one to guarantee stability in the two-dimensional case is the existence of a function $H$ such that $X=\frac{\partial H}{\partial y}, Y=-\frac{\partial H}{\partial x}$. If this condition is fulfilled, Levi-Civita added, neither the criterion of stability nor that of instability are qualitative. The same holds for the equilibrium of a material system under conservative forces. But what happens-he asked himself-when we move from a study of the stability of equilibria to a study of stability of movements? The use of the section method is essential in this transition and Levi-Civita started to study the neighborhoods of periodic solutions employing a qualitative approach to investigate their stability.

\footnotetext{
38 This work contains results of three publications from the previous year, as explained in Dell'Aglio and Israel (1989).
} 
Before describing how he did that, we emphasize that it is one of the first times we find a clear criterion for making a distinction between a qualitative and a quantitative approach. While the latter concerns an explicit search for analytic expressions, the former is satisfied with the implicit verification of certain conditions by means of inequalities, or features that can be inferred directly from the set of solutions seen as movements (without any necessity for providing an analytic expression). Lyapunov had already worked with a criterion of this kind, but did not put forward a discussion about its qualitative character.

In order to investigate the stability of a periodic solution, Levi-Civita used Poincaré's section method, and concluded that "tutto si trova in tal modo ricondotto allo studio delle transformazioni puntuali" (Levi-Civita 1901, p. 223). The novelty of his approach was to define stability by means of the behavior of a point transformation $T$, defined over the section, in the neighborhood of the fixed point $O$ corresponding to the periodic solution (Fig. 1). Such transformation is unstable whenever positive and negative iterations of a point $P$ by $T$ escape any given neighborhood of $O$, no matter how small; and $T$ is stable in the opposite case. The periodic solution associated to a fixed point $x_{i}=0$ :

Will be said stable (...) if and only if, for each arbitrarily small neighborhood $E$ of the origin $\left[x_{i}=0\right]$, there exists an $H$ such that, taking in $H$ the initial position of the moving point, it remains in $E$ for all positive and negative values of $t$. There will be instability in the opposite case, that is to say if does not exist an $H$ endowed with the cited property; or, in other words, if arbitrarily close to the origin, there always exist an initial position $P_{0}$ from which the moving point can be, for at least an instant, out of $E$ (Levi-Civita 1901, p. 262). ${ }^{39}$

Two pages later he concluded:

The question of stability of periodic solutions reduces to that of point transformations(Levi-Civita 1901, p. 264). ${ }^{40}$

He then investigated the properties of the point transformation $T$, in particular the case in which it is unstable, using a result extracted from (Lyapunov 1897). In this point, Levi-Civita had already defined stability, without attributing his definition to Lyapunov. But the way he used Lyapunov results shows they must have had the same conceptions of stability. ${ }^{41}$

\footnotetext{
39 Sarà a dirsi stabile (...) allora e solo allora che, per ogni intorno comunque piccolo $E$ dell'origine $\left[x_{i}=0\right]$, ne esiste um $H$ tale che, prendendo in $H$ la posizione iniziale del móbile, questo rimane in $E$, per qualunque valore positivo o negativo di $t$.

Vi è instabilità nel caso opposto, ossia se non esiste um $H$ dotato della anzidetta proprietà; o in altri termini se, vicino quanto si vuole all'origine, esiste sempre qualche posizione iniziale $P_{0}$, a partire dalla quale il móbile si trova, in un istante almeno, fuori di $E$.

40 La questione della stabilità delle soluzioni periodiche si riduce a quella delle transformazioni puntuali.

41 In fact, Levi-Civita's definition is different from Lyapunov's at a single point: it considers not only future stability, but a the past stability of a solution as well. In mathematical terms, it is an irrelevant difference since it is equivalent to a mere change of $t$ for $-t$ in the solution. Barrow-Green (1997, p. 182) notes that this addition may reflect Levi-Civita's concern with classical mechanics, as his definition reflects the reversibility of physical processes.
} 
For a system of equations:

$$
\frac{\mathrm{d} x_{i}}{\mathrm{~d} t}=X_{i}\left(x_{1}, x_{2}, \ldots, x_{n}\right)
$$

where $i=1, \ldots, n$, Lyapunov considered each $X_{i}$ a linear function of $x_{i}$, added to a residue of second order (or higher):

$$
X_{i}=p_{i 1} x_{i}+\ldots+p_{i n} x_{n}+R_{i}
$$

The equilibrium solution $x_{i}=0$ was defined to be stable or unstable exactly in the same manner as in his second thesis. He showed that it is unstable if there are eigenvalues of the $\left[p_{i j}\right]$ matrix with positive real part.

A similar method was used in Lyapunov (1907) in order to analyze the case of eigenvalues with negative real parts, which allows one to infer stability (and also to justify the linear approximation for small perturbations). In fact, when the equation has constant coefficients, the linear approximation is sufficient for a study of stability in almost all cases, except when eigenvalues have a zero real part, i.e., when they are purely imaginary. Levi-Civita analyzed precisely this situation.

If not all the characteristic values associated with $T$ have absolute value 1 -which means that the characteristic exponents are pure imaginary-one can solve the problem of stability. As Poincaré had demonstrated in (Poincaré 1892-1899), the case where all characteristic values have absolute value 1 corresponds to stability, but this conclusion is only valid in the first approximation. It is necessary to see if Poincaré's method is still efficient for higher order approximations. Levi-Civita showed that the demand that characteristic exponents be purely imaginary (stability in the first approximation) is far from assuring stability of a periodic solution in any approximation.

The article "Sopra alcuni criteri di instabilitá" follows with the consideration of canonical equations:

$$
\frac{\mathrm{d} p_{i}}{\mathrm{~d} t}=-\frac{\partial F}{\partial q_{i}}, \quad \frac{\mathrm{d} q_{i}}{\mathrm{~d} t}=\frac{\partial F}{\partial p_{i}}
$$

where $i=1,2$ and $F$ does not depend on $t$. For a periodic solution, examined by means of a point transformation over the section, Levi-Civita concluded that it is essential to know whether the perturbed trajectory returns infinitely close to the fixed point or recedes from it after an arbitrarily small variation of the initial condition. It is desirable to show, according to him, the general unstable character of the transformation $T$, even when the characteristic exponents are purely imaginary (condition used by Poincaré to define a stable periodic solution).

Levi-Civita thus conjectured that the general case is unstable and demonstrated this fact for the specific situation obtained when the average movements of the sun and the moon around the earth are commensurable. This means that the case considered as "stable" by Poincaré can be unstable if the precision of the approximation is increased. Hence, it becomes clear that Levi-Civita's definition of periodic solutions stability fits better into the qualitative approach than that proposed by Poincaré. 
Using the section method, Levi-Civita showed that there are zones of instability that condense around the orbit of the planet. ${ }^{42}$ What remains to be discovered is how those regions distribute themselves inside the domain. But the author admitted that his methods did not allow him to go very far into this question, not even to know whether the instability zones cover the whole plane or not (Levi-Civita 1901, p. 295). Birkhoff started from this point in his own studies about the problem.

\subsection{Tradition and innovation in Levi-Civita's works}

In their analysis of Levi-Civita's style, Dell'Aglio and Israel (1989) have already noticed that his works are in some ways very singular, in particular because they reconcile the qualitative viewpoint with the traditional approach of Lagrange's analytical mechanics. According to them, what puts Levi-Civita and Lyapunov's definitions together is the fact that both mathematicians made direct use at the start of their work of Lagrange's and Dirichlet's definitions of stability, with a view to adjusting them to more general problems.

For these historians, one of the main features of Levi-Civita's style is this effort to reconcile tradition and innovation, without marking out points of rupture and without even emphasizing his own original contributions. In fact, throughout his article on the stability criteria, he never emphasized the difference between his definition for the stability of periodic solutions and the one proposed by Poincaré.

Although Levi-Civita's methods seem innovative, Dell'Aglio and Israel remark that his attitude is typical of someone who believes in a line of continuity within the traditional methods. This can be understood in terms of his conservative belief that a scientific domain must pay tribute to already accumulated results.

These traits in Levi-Civita's scientific personality contrast sharply with Birkhoff's. The contribution of the latter to the constitution of the domain of dynamical systems consists precisely in establishing criteria that identify what is new in qualitative methods, explicitly showing where and how they converge or diverge by comparison with the traditional approach.

This difference between Levi-Civita and Birkhoff's styles can be felt in the letters they exchanged from 1913 onwards. ${ }^{43}$ In one of the first letters, dated March 3rd 1913, Birkhoff confessed how Levi-Civita's study of stability had impressed him as almost the only definite advance in the restricted three body problem made to that time. The general keynote of the letters was that of mutual respect and admiration in face of the research progress each one had accomplished. After 1918, the discussion on issues in celestial mechanics became briefer.

\footnotetext{
42 This example eliminates, according to Levi-Civita, the expectation that stability is less exceptional in concrete cases than it is in the abstract mathematical case. In his view, it is rather surprising that stability appears as an exception when, from an astronomic point of view, everything points to the opposite idea (Levi-Civita 1901, p. 224).

43 Birkhoff's letters to Levi-Civita have been published as (Nastasi and Tazzioli 2000, pp. 199-220). LeviCivita's letters to Birkhoff have not been published yet. I thank Rossana Tazzioli for her kind help in this matter.
} 
One of the last letters on the three body problem is from October 26th 1918. It is Birkhoff's response to Levi-Civita's response to his paper entitled "Sur la régularization du problème des trois corps"(Levi-Civita 1920). For the American mathematician, this work represents the definitive treatment of the regularization problem, but he found it strange that Levi-Civita did not seem interested in showing that the main results of Sundman could be deduced from it. ${ }^{44}$

On December 1st Levi-Civita replied to the letter saying that Sundman's famous results are immediate corollaries of the point already established in his own article:

I have the impression that this is a true pedagogical detail, noticeable afterwards, and summing up, without changing anything in the concept, the final considerations of Mr. Sundman. That is the reason why I neglected it. ${ }^{45}$

We will return to this correspondence later. So far, reference is made to this response only to highlight the differences between Levi-Civita's and Birkhoff's styles and general positions. ${ }^{46}$ Birkhoff considered it strange that Levi-Civita did not emphasize the fact that a known result can be inferred in a new, simpler and clearer, manner. As we will see next, for Birkhoff it was fundamental to clarify the essential difference in methods, even if they end up with the same results.

\section{A clear criterion for distinguishing qualitative and quantitative approaches to stability}

The name of Birkhoff first appeared in the international mathematical community in the 1910s and one of his first texts on dynamical systems is the article "Quelques théorèmes sur le mouvement des systèmes dynamiques" (Birkhoff 1912). At the very beginning, Birkhoff defined the so-called "recurrent" movements, which are those that arbitrarily approximate any of its positions, past and future. Such recurrent movements are important inasmuch as they have a special property of representing, in a satisfactory manner, the entire extension of the movement, to any degree of approximation, during the whole period of time. Equilibrium points and periodic movements are particular cases of recurrent movements.

Birkhoff's recurrent movements are Poisson stable. It is precisely the denomination of "stability" for the recurrence property that he had criticized in 1935: "this [Poincaré's] use of the word 'stability' is, however, unfortunate" (Birkhoff 1935, p. 310). He then noticed that it is more convenient to keep the term "stability" for other properties:

The fundamental fact to observe here is that this concept [stability] is not in itself a definite one, but it is interpreted according to the question into consideration

(Birkhoff 1935).

\footnotetext{
44 The works of Sundman had appeared some years before (Sundman 1912).

45 J'ai l'impression qu'il s'agit d'un véritable détail didactique, réalisable après coup, en résumant, sans y changer rien en concept, les dernières considérations de M. Sundman. C'est pour cela que je me suis passé. 46 A comparison between Levi-Civita's and Sundman's works on the three body problem can be found in Dell'Aglio (1993).
} 
Between the 1912 article and the comment quoted above stability was very often discussed by Birkhoff. We will see just some examples, more directly concerned with the stability of periodic solutions and the definition of a qualitative treatment.

\subsection{Periodic solutions and point transformations}

One of Birkhoff's first important results was the demonstration of Poincaré's last geometric theorem (Poincaré 1912). It was a proposition according to which the existence of an infinite number of periodic trajectories in dynamical problems, such as the restricted three body problem, could be inferred from the analysis of a transformation of a ring to itself (defined between two closed curves over the surface of a two-dimensional section).

In 1912, Poincare had put forward the hypothesis that, if an area preserving transformation $T$ takes the ring to itself, in which the circles forming the boundary move in opposite angular directions, it is possible to conclude that there are, at least, two invariant points inside this ring. By using the section methods, a three-dimensional periodic trajectory can be reduced to an invariant point over the section, and if we trace out a ring around that point, the result allows one to conclude that there are at least two other periodic trajectories in the neighborhood of the first one. As Poincaré noted in particular cases the demonstration of this theorem is topological in nature, but it fell to Birkhoff to find, in 1913, a simple procedure capable of ensuring the result (Birkhoff 1913). It suffices to apply successively the transformation $T$ (or $T^{-1}$ ) noting that the mere fact of preserving areas can be used to conclude that a stripe of the ring should be taken to another stripe (with the same area), which necessarily intercepts the first one. After successive iterations of the transformation, the intersections of multiple rings should have a common point.

After publishing his paper in 1913, motivated by the usefulness of a two-dimensional point transformation, Birkhoff devoted his studies to dynamical systems with two degrees of freedom. This culminated in the publication of a paper on the subject (Birkhoff 1917), in which he stated that these systems are the "simplest type of non-integrable dynamical problems and possess a very high degree of mathematical interest"(Birkhoff 1917, p. 1).

In this article, Birkhoff stressed Poincaré's section method. He quoted Levi-Civita's article of 1901 to show the importance of point transformations and to present a definition of stability and instability in terms of its properties. By studying the effects of a two-dimensional transformation on a surface of a definite genus, he managed to establish a relationship between this number and the number of invariant points that are stable and unstable. In addition to that, Birkhoff showed that, given some conditions on the surface, there are infinitely many periodic orbits.

In the following years, he continued to work on this problem and, in his correspondence with Levi-Civita, he seemed surprised by the possibility of obtaining a positive answer for a typical problem of stability. But this requires that the definition of stability in use be stated clearly. On 1 May 1918 he wrote:

At present I am at work with the paper which forms the natural conclusion to the earlier one [from 1917] (...) and I have apparently obtained some results which 
I have been altogether astonished at. In particular I believe that I will be able to demonstrate that the periodic orbits of the simplest type in the restricted problem of three bodies are stable in the true sense that, if a certain number is irrational, nearby orbits remain nearby, whereas, if it is rational, nearby orbits remain within a fixed neighborhood of the periodic orbit, although this neighborhood is not 'infinitesimal'. I have not yet verified all of the analytic details involved in this particular application of the general methods of my paper, which I expect to finish in a couple of months. The result as I have stated it is in harmony with your own fundamental results on instability, but nevertheless it has gone contrary to my preconceived ideas (Nastasi and Tazzioli 2000, p. 206). [our italics]

Here the definition of stability "in the true sense" emerges, which means that solutions near the periodic orbit remain nearby. The article in preparation that Birkhoff referred to was published in 1920 and became one of his most famous: "Surface transformations and their dynamical applications" (Birkhoff 1920b). Here, he presented a rigorous definition of the section method and analyzed several properties of point transformations defined in the neighborhood of an invariant point.

The use of the section method, and the resulting reduction of dynamical problems to problems about point transformations, is one of the main steps in the institutionalization of dynamical systems as a new domain. The quotation above is one of the first occasions showing Birkhoff's desire to distinguish a new field, which is the question we will treat in our conclusion. Before arriving at this point, we must explain his results on the stability of periodic solutions.

\subsection{The instability of Poincaré's stable periodic solutions}

In order to undertake a general study of point transformations, Birkhoff started with a classification of invariant points. If $u_{1}$ and $v_{1}$ are coordinates of a transformed point, the transformation $T$ is written as power series of $u$ and $v$, with real coefficients:

$$
\left\{\begin{array}{l}
u_{1}=a u+b v+\ldots \\
v_{1}=c u+d v+\ldots
\end{array}\right.
$$

The classification of the invariant points is based on the linear terms in those series and depends upon the nature of the roots of the characteristic equation:

$$
\rho^{2}-(a+d) \rho+a d-b c=0
$$

where $a d-b c=1$ and the roots are $\rho$ and $\frac{1}{\rho}$.

In the first case, if $\rho$ is real with a numerical value not equal to unity, a point $P$ in the surface will move on a hyperbola after successive applications of $T$ or $T_{-1}$. In the third case considered, this point would move along a pair of parallel lines.

But the most important for us is the second case, in which $\rho$ is complex and of modulus 1. The transformation is a rotation around the invariant point through an angle $\theta$ and every point $P$ remains at a fixed distance from the invariant point after successive applications of $T$ or $T_{-1}$. This case can be divided in two subcases: the 
Fig. 4 Ring of instability-hyperbolic points between two invariant curves $C_{1}$ and $C_{2}$ around a fixed point $P$

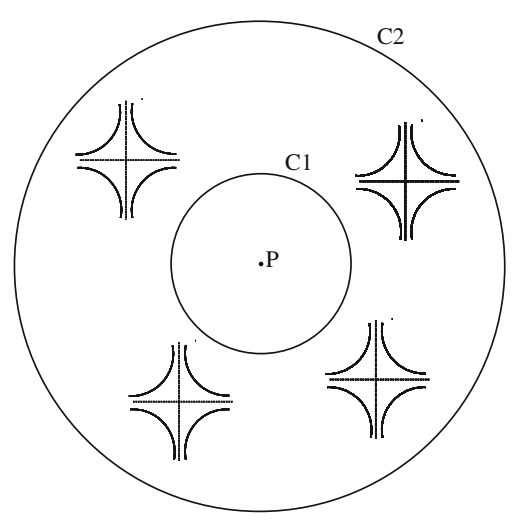

irrational (when $\frac{\theta}{2 \pi}$ is irrational); and the rational (when $\theta=0$ or $\frac{\theta}{2 \pi}=\frac{p}{q}$, and this fraction is not an integer).

Birkhoff added that the essence of this distinction is brought out clearly by means of a stability definition, explicitly referred to as being as analogous to the one given by Levi-Civita in 1901:

If a neighborhood of an invariant point can be so taken that points arbitrarily near the invariant point leave this neighborhood after successive applications of $T$ or $T_{-1}$, the invariant point is unstable; in the contrary case the invariant point is stable (Birkhoff 1920b, p. 5).

The determination of curves on the surface that remains invariant under successive applications of $T$ or $T_{-1}$ is essential for Birkhoff's method. If there are curves of this sort, given by real series (analogous to the curves $K$ and $K^{\prime}$ already described by Poincaré), the transformation is called hyperbolic. In the contrary case it is called elliptic.

The first case, of real $\rho$, is the prototype of a hyperbolic situation; whereas the irrational situation is elliptic, since we have a pair of conjugate imaginary invariant curves. The other ones may be either hyperbolic or elliptic. Birkhoff explained the connection between the hyperbolic-elliptic distinction and the question of stability. The stable case must be defined by the existence of an infinite number of invariant closed curves surrounding the invariant point (Birkhoff 1920b, p. 80).

From now on, we will just treat the case when the roots are imaginary of modulus 1 , defined as stable by Poincaré. This situation is regarded now as the one furnishing a possibility of stability, but not necessarily stable.

Birkhoff showed that in the ring formed by two invariant curves $C_{1}$ and $C_{2}$ there may or not be other invariant curves. If there are, this ring can be subdivided further into similar rings. In the integrable case, these rings reduce to single invariant curves, but in the general case they can be viewed as "rings of instability" (Birkhoff 1920b, p. 91). Inside those regions there can be hyperbolic points with very complex behavior (Fig. 4).

The last six pages of his more than one-hundred-page paper give some dynamical applications of these methods. Birkhoff quoted several connections with celestial 
mechanics all over the text, but his work is more concerned with a general study of point transformations defined in the neighborhood of an invariant point. He closed the paper by saying that the remarkable diversity and complexity of structures emerging when we analyze the solutions of dynamical systems with two degrees of freedom is not yet well understood, and the theory of point transformations must help one to grasp what is going on.

Levi-Civita had already demonstrated the instability of a periodic solution for a system where the mean motions of the planetoid and the two other bodies are commensurable. This occurs because there are solutions that approach and recede from the periodic solution. In Birkhoff's language, this means that there are hyperbolic points in the neighborhood of an invariant point.

If the invariant point is hyperbolic, there are invariant curves that go through it: one curve on which the iterated points recede from the invariant point (expanding dynamics _- responsible for instability — and another one on which the iterated points approach the invariant point (contracting dynamics). Birkhoff (1920b, p. 100) showed that if these two sets intersect, there is an infinite number of intersections, which makes the trajectories' dynamics extremely complex. In fact, the hyperbolic points are doubly asymptotic in the sense that Poincaré had already announced in 1890. As he had already noted, the presence of this kind of points, also called "homoclinic", makes the dynamics extremely complex. ${ }^{47}$ In the figure above, the hyperbolic points in the ring of instability between curves $C_{1}$ and $C_{2}$ can be points of this type, and we find an explanation there of the difficulties mathematicians had found in studying this problem since Poincaré.

Despite that one can expect that stability subsists, as those points are entirely contained in the rings of instability, i.e., they are confined between closed invariant curves. This conclusion will lead to the main definition put forward by Birkhoff for the stability of periodic solutions. The possibility that instability remains confined was celebrated by Levi-Civita in his response to the letter quoted before, in which Birkhoff informed him on his progress during the 1920 article preparation. On June 3rd 1918, Levi-Civita wrote:

My first essay - in which instability in the strict sense emerged as a general rule - was rather disappointing. Even more striking are the news about the successful outcome of your brilliant and insightful researches that enabled you to grasp the laws of distribution of periodic solutions, and also to deduce from them that, in any case, they are stable in a suitable and very clear sense of this word. ${ }^{48}$

In October 26th, Birkhoff replied with the following:

Although I have not yet had the leisure to give my recent work on stability the extraordinarily careful verification it demands I am reasonably confident of the

\footnotetext{
47 On the history of the discovery of doubly asymptotic solutions by Poincaré see (Anderson 1994) and (Barrow-Green 1997).

48 Mon premier essai, d'où ressortait comme règle génerale l'instabilité au sens strict, était plutôt déourageant. D'autant plus frappante est la nouvelle de l'heureuse issue de vos géniales et pénétrante recherches, qui vous ont permis de saisir les lois de distribution des solutions périodiques, et d'en déduire qu'en tout cas elles sont stables dans une convenable et bien nette acception de ce mot.
} 
following critical theorem: Let $C_{1}, C_{2}$ be two distinct continuous closed curves about a fixed point $\mathrm{O}$ which are met by any half-line ending at $O$ in one and only one point. Let $\mathrm{T}$ be a direct, continuous, one-to-one area-preserving transformation of the area between $C_{1}$ and $C_{2}$ into itself, which has the further property of taking half-lines ending at $\mathrm{O}$ into curves met by any half-line ending at $\mathrm{O}$ at most once. There will then exist an invariant continuous closed curve $C$ about $O$ met by any half-line ending at $O$ in one and only one point. From this theorem follows at once the existence of an infinitude of such invariant curves, which need not however fill up the region between $C_{1}$ and $C_{2}$ (Nastasi and Tazzioli 2000, p. 209).

In the integrable case, there are only invariant closed curves in the neighborhood of the invariant point. This is equivalent to complete stability (i.e., all the solutions in the neighborhood of a periodic solution are situated on invariant tori over which the integral is constant). Birkhoff was saying, in other words, that stability in the non-integrable case corresponds to the existence of invariant closed curves enclosing the invariant point, even though there may be hyperbolic points between two of these curves, as in Fig. 3. In fact, for a non-integrable system very close to an integrable one, Birkhoff demonstrated that there may be doubly asymptotic hyperbolic points in the rings of instability, but it does not disturb stability, at least in the sense he defined it. 49

This way to enunciate the problem became more precise after 1925, when Birkhoff proposed an extension of Poincaré's last theorem (Birkhoff 1925). By means of a topological procedure ${ }^{50}$ he showed that in the neighborhood of an elliptic periodic movement there are an infinite number of periodic movements. ${ }^{51}$

As was said previously, an invariant point is considered stable if there is an invariant closed curve enclosing this point, as that implies the confinement of other trajectories in its neighborhood. Birkhoff then studied the nature of all possible invariant curves near an invariant point over the section, and demonstrates that an elliptic periodic movement is a "cluster motion" of an infinite number of other periodic movements. The method allows another conclusion: there are an infinite number of stable and unstable periodic movements in the neighborhood of an elliptic periodic movement. ${ }^{52}$

In the integrable case, an elliptic fixed point must be stable. This coincidence led Poincaré to define the stability of a periodic solution in terms of the conditions now used to characterize ellipticity: pure imaginary characteristic exponents. Birkhoff's results imply that, for a system obtained by a very small perturbation of an integrable one, an elliptic fixed point may be unstable.

\footnotetext{
49 These results are in the beginning of the now called KAM Theory, in a reference to mathematicians Kolmogorov, Arnold, and Moser.

50 Birkhoff noticed that the importance of Analysis Situs to study the types of periodic movements is clarified with Morse's (1925) works.

51 It is even possible to eliminate the condition that transformation $T$ preserves the areas (Birkhoff 1925, p. 299).

52 Apart from the existence of non-periodic movements that are uniform limits of periodic movements, and therefore "quasi-periodic".
} 
The necessary and sufficient condition to ensure stability is the existence of an infinite number of invariant closed curves arbitrarily near the invariant point. This is the same as saying that the periodic movement is stable if there exists an infinite number of invariant tori closing down upon the curve of the given periodic motion. This is the true sense of stability Birkhoff had referred to in his 1st May 1918 letter to Levi-Civita.

Hence, for a non-integrable situation, even if close enough to an integrable one, the discovery of complex movements in the neighborhood of an elliptic periodic point demands a redefinition of stability. That is the reason why a periodic movement is said to be "stable" only if movements sufficiently close to the periodic movement remain in a small neighborhood at any instant, as it appears in the 1927 book Dynamical Systems. Birkhoff stated accordingly that:

Stability in this fundamental qualitative sense is not to be confused with the 'complete formal stability' introduced earlier, and a periodic motion 'of stable type' may or may not be stable (Birkhoff 1927a, p. 220).

The condition used by Poincaré to define stability of a periodic solution only indicates whether or not the invariant point, to which the solution corresponds over the section, is of "stable" type. The two situations-stable and of stable type-are equivalent for an integrable system, since in the neighborhood of an elliptic invariant point on the section there is a family of invariant closed curves arbitrarily close to the invariant point. However, in the non-integrable case, this equivalence is no longer valid.

\subsection{Several types of stability}

Levi-Civita had already distinguished qualitative and quantitative stability. Birkhoff made the definitions more precise. In his view, we can speak of two different kinds of stability: one that can be named "formal" and another one that is properly speaking "qualitative". The formal definition reflects the classical treatment about the problem of stability of the solar system since Lagrange and Laplace. Stability is verified by means of analytic conditions expressing properties of solutions related to periodicity. The qualitative definition has a perturbative character and considers stability as a property of the whole set of solutions seen as movements.

Both types can be found in Poincaré's works. His definitions of stability for periodic solutions reflect properties of series developments. The inspiration for the definition of Poisson stability comes from traditional approaches, but the methods Poincare used to investigate it were quite geometrical — as the intervention of the recurrence theorem in Poincaré (1892-1899). On the other hand, stability as confinement can be said to be qualitative.

In the analysis of the stability of periodic solutions, we can also identify mixed approaches: the stable case is defined in a formal manner, but the unstable one, defined as opposed to the stable, is analyzed by means of qualitative methods, as surfaces without contact and asymptotic surfaces. Poincaré showed that, in the three body problem, when the average movement of the small body is commensurable with the average movement of the two other, formal series can be obtained and there is stability in the traditional astronomic sense (formal). In the same commensurable case, by using a 
better than linear approximation, Levi-Civita showed that a periodic solution is not stable in the sense he designated as "qualitative". As a consequence, formal stability does not imply qualitative stability.

This conclusion and the research described in the previous part led Birkhoff to distinguish two types of stability in the book Dynamical Systems (Birkhoff 1927a). On the one hand one has the "formal" definition, named "astronomic" or "trigonometric". On the other, there is a properly qualitative definition, also named "mathematical" or "perturbative". The formal condition is used to designate an invariant point of "generally stable type". This point is properly "stable" when all movements going through initial conditions sufficiently close remain indefinitely in a small neighborhood of this point.

The definition proposed by Levi-Civita in this case is, according to Birkhoff, qualitative par excellence. Birkhoff briefly quoted Lyapunov's research in (Birkhoff 1927a, p. 122), without emphasizing he had already proposed a stability definition of the same kind. We can say that the use of Levi-Civita's works shows an indirect influence of Lyapunov. However, it is important to notice that Birkhoff did not take from Poincaré the same tools as Lyapunov had done. For him, the section method, which did not interest Lyapunov, is the most fruitful invention presented by Poincaré in his works on differential equations. This indicates a different perception of the importance of perturbative stability, even if Birkhoff's definition is analogous to Lyapunov's.

Birkhoff added that an "outstanding question in dynamics is whether or not the complete formal stability assures stability in the fundamental qualitative sense" (Birkhoff 1927a, p. 227). From this moment onwards, one needs to answer the following general questions: Does formal stability ensure such qualitative stability (which Birkhoff calls "actual stability")? If not, does formal stability imply actual stability in important special cases such as the restricted three body problem?

Birkhoff stated then that a movement has "stability of the $m$ th order" when both properties are satisfied:

(A) movements in the neighborhood of the given movement remain within a controlled distance according to $\frac{1}{\epsilon^{m}}$ (perturbative stability);

(B) the coordinates of any movement can be represented in terms of trigonometric series with an error limited by $\epsilon^{m}$ during a fixed interval (trigonometric stability).

A movement is "completely stable" if it is stable to all orders. Perturbative stability does not need trigonometric stability (B), although this is sufficient for a demonstration of perturbative stability in some cases (B implies A, but A does not imply B). The condition (B) is, therefore, stronger and often used to infer (A), even if it is harder to obtain than (A). Hence, perturbative stability may be present even if one is unable to write the series expansion of the movements. In this case, it is worth investigating directly, by means of geometrical and topological methods, whether there is perturbative stability or not.

Before Birkhoff, the two notions of stability expressed by conditions (A) and (B) had been mixed up. One of the reasons for that lies in the fact that these conditions are equivalent for the equations of dynamics in the integrable case. Research done until Birkhoff's time was concerned essentially with the nearly integrable situation. However, in the non-integrable case, conditions (A) and (B) are different and it is 
necessary to propose new methods to investigate qualitative stability directly in the space of motions.

In the second half of the 1920s Birkhoff wrote articles in which he declared his intention of unveiling the "mysterious significance of the Hamiltonian equations". ${ }^{53}$ In these works, Birkhoff distinguished quantitative and qualitative stability in the "general equations of dynamics"-equations in Hamiltonian form that arise from a special type of variational principle and describe a nearly integrable system. He suggested that, in order to understand the relevance these equations acquired in the nineteenth century, one must have in mind there is a special relationship between formal complete stability and qualitative stability in the systems they describe. We will not take time to examine these works because this would lead us away from our goal. Birkhoff wished to show that the importance of these equations is less a consequence of the natural laws than a consequence of the fact that, for them, the two types of stability (formal and perturbative) are indeed associated. However, in order to investigate the stability of solutions defined by non-integrable Hamiltonian systems one has to describe the behavior of their trajectories in the space of motions. This same procedure should be developed for a general, not necessarily Hamiltonian, system.

\section{Conclusion: stability and dynamical systems theory}

The qualitative methods introduced by Poincaré are now unanimously celebrated as a great invention. Notwithstanding, it was not always like this. Regarding the paper that earned the prize offered by the King of Sweden, Barrow-Green observes that:

Its sheer novelty would probably have been sufficient to make the memoir almost inaccessible to those of a more practical persuasion. This was certainly the view adopted by Mittag-Leffler, who, while studying the original memoir, expressed the concern that Poincaré's resolution of the restricted three body problem was given in a form that would be difficult to understand by anyone except those familiar with this work (Barrow-Green 1997, pp. 107-108).

From the end of the nineteenth century up to the first two decades of the twentieth century, it seems that qualitative methods were not very easily accepted. In an article published in the journal Science, Birkhoff (1920a) ranked the most important results about dynamical systems since the very end of nineteenth century, making a vehement defence of qualitative methods. In the final part of the article, he argued that qualitative information about the movements defined by a dynamical system is fully satisfactory:

The recent advances supplement in an important way the more physical, formal, and computational aspects of the science by providing a rigorous and qualitative background.

To deny a position of great importance to these results, because of lack of emphasis upon the older aspects of the sciences would be as illogical as to deny the importance of the concept of the continuous number system merely because of

\footnotetext{
53 We refer to the texts Birkhoff (1926b), Birkhoff (1926a), Birkhoff (1927a) and Birkhoff (1927b, p. 1).
} 
the fact that in computation attention is confined to rational numbers (Birkhoff 1920a, p. 5).

It seems there had been researchers who questioned the relevance of qualitative analysis. Even if it is an interesting question, it is not our purpose in this article to investigate to whom the above statement was directed. But seemingly in 1920 there was a need to speak in favor of qualitative methods.

Some years earlier, Karl Sundman had solved, in a certain sense, the three body problem. By showing that there exists a convergent series expansion for the coordinates of the bodies valid for all time values, he had provided an analytic solution to the problem, which seemed impossible to many mathematicians before him. Two papers on this problem were published respectively in 1907 and 1909 in the Acta Societatis Scientiarum Fennicae [(Sundman 1907) and (Sundman 1909)], but Sundman's works only became known after the publication of his main results in the Acta Mathematica, (Sundman 1912).

The historical context of these works and their reception have been analyzed in detail by Barrow-Green (2010). She observes that in the years following the 1912 publication, "while Sundman's mathematical dexterity was widely lauded, it had not gone unnoticed that his result gave no practical help to astronomers, whose agenda was rather different from that of the mathematicians" (Barrow-Green 2010, p. 191). Some mathematicians doubted that Sundman's series could be suitable for providing qualitative information about astronomical phenomena than the trigonometric series, as these series give no properties of the motion. In 1917 Whittaker referred to the result in the new edition of his Treatise on the Analytical Dynamics of Particles and Rigid Bodies (Whittaker 1917) but, as Barrow-Green notices, described it as a considerable advance rather than a solution, indicating that he was hesitant about how to classify the result. This ambivalent reaction is characteristic of what she describes as the third stage in the reception of Sundman's results, which began after the First World War and continued up until the early 1990s. In this period, Poincaré's work had changed the nature of the subject, at least in the highly theoretical sense, and it was not obvious in which direction the research on celestial mechanics would go. Barrow-Green points out that:

Poincaré's mathematics was difficult and held little immediate promise for the practical mathematical astronomer. Sundman's solution was in a similar vein. While not of the same level of difficulty as the work of Poincaré, it nevertheless encompassed an analytical result: a result in pure mathematics and without practical application. For applied mathematicians the problem remained ostensibly unsolved and their appreciation of Sundman's achievement was correspondingly muted (Barrow-Green 2010, p. 195).

The discussions about the status of Sundman's results raised the question of what counts as a "solution" in the context of an applied mathematics problem, as BarrowGreen remarks, but the same question can be brought to the context of pure mathematics. In the article cited above, published in Science, Birkhoff qualified Sundman works as "remarkable", but he added immediately after that his results "solves" the problem 
of three bodies in a highly artificial sense (Birkhoff 1920a, p. 53). ${ }^{54}$ In the same 1920 article, Birkhoff states that the question of stability is beyond the demonstration of stability in celestial mechanics. Rather, it is a question of pure mathematics, that of determining the general character of the limitations upon the possible variations of the coordinates in dynamical problems (Birkhoff 1920a).

This brings us to the heart of the qualitative approach. As Birkhoff explained, the question of stability must be seen as a subproblem of another, more general problem: to describe the global aspect of the set of trajectories in the space of motions. In other words, it is necessary to describe the qualitative aspect of trajectories as a whole in order to obtain some information about stability.

Poincaré had already performed an analysis of the same kind. An example can be found in the first parts of "Sur les courbes", when he described the topographic aspect of the whole set of trajectories in a two-dimensional situation to infer results about stability. In higher dimensions, we can often find similar approaches in his works about celestial mechanics. However, the qualitative definition of stability that intervenes in these results - the one that associates it with the confinement of trajectories in some region of space-remains almost implicit. Unstable situations can be identified by means of properly qualitative methods, but the stability definition in Poincaré's works is still dependent upon the possibility of writing functions, what characterizes formal stability.

We hope to have shown the role qualitative analysis has played in the constitution of dynamical systems as a new domain of research, and also the central place of stability in this history. From the moment treatises and popular papers begin to appear, such as those published by Birkhoff, we can say a new field of research has emerged. Definitions thus become clearer and, from this new viewpoint, the older definitions having similar characteristics to the ones then proposed are considered "implicit". In this sense we can describe Poincaré qualitative stability conception as being merely implicit.

Only with Levi-Civita's and-above all-Birkhoff's works, both making strong use of Poincaré's methods, does one come to an explicit qualitative definition of stability. The issue of periodic solutions provides an excellent example of how things happened. In the integrable case, the elliptic situation is stable. But beyond that case, even if the system is very close to an integrable, it is still necessary to distinguish stability from ellipticity. As a consequence, it is also necessary to propose a definition of stability "in the true sense", as quoted in the letter Birkhoff sent to Levi Civita in 1918 (mentioned in Sect. 6.1).

In the non-integrable case, qualitative stability cannot be studied by means of analytical expressions and a direct analysis of the possible types of solution in the neighborhood of periodic movements is needed. Hence, it is important to investigate the invariant curves that goes through the fixed point on the transverse section. The new criterion of selection, fundamental to research to today, respects the separation between

\footnotetext{
54 Barrow-Green notices that this was not Birkhoff's last pronouncement on the subject, since he devoted a large part of the final chapter of his book Dynamical Systems to Sundman's contribution. The discussion we propose here being not that of Sundman's works reception, we do not think it is worth to analyze the commentaries.
} 
elliptic and hyperbolic situations. ${ }^{55}$ This classification gives room for the description of the whole set of trajectories in the space of motions. But the remarkable diversity and complexity of structures of trajectories defined by dynamical systems is very difficult to grasp, and one of Birkhoff's main contributions of is to have suggested that a theory of transformations can be helpful for this task.

The context of non-integrable Hamiltonian systems, and even the more general systems investigated by Birkhoff is another factor that has led research in the direction of a general description of trajectories. The richness, and above all the inexorability in the use of qualitative methods could not appear while the search for integrability, in the classical meaning of the term, remained the main goal of research. When it is possible to calculate and write analytic expressions, the qualitative methods are merely auxiliaries. Yet, when those analytical methods prove to be insufficient, it becomes necessary to search for new directions and the qualitative approach assumes a central place.

From his first articles, Birkhoff insisted on the importance of Poincaré's works for an inquiry into non-integrable systems. Beyond the studies of integrable systems undertaken by Lagrange, a thorough investigation of non-integrable dynamical problems is essential for the further progress of research (Birkhoff 1915). And he continued:

Until now only periodic movements and certain closely allied movements have been treated with any degree of success in such problems, but the final goal of dynamics embraces the characterization of all types of movement, and of their interrelation (Birkhoff 1915).

Here, it is possible to notice his concern with the general study of trajectories in relation to the consideration of non-integrable systems. In 1934, Birkhoff reaffirmed this connection in an analysis of Poincaré's works on differential equations:

They [the works of Poincaré] are likely to stand always as the most important works in this field [celestial mechanics]. Lagrange, Jacobi, and other mathematicians had fastened attention upon integrable cases (...) In the field of celestial mechanics, Laplace and the succeeding theoretical astronomers had been content to use formal series as a means of systematic computation. But Poincaré was the first to attack the general question of non-integrable systems of differential equations from a purely mathematical point of view (Birkhoff 1934).

The qualitative approach is thus recognized as being "purely mathematical". In the domain of differential equations, mathematics has to progress beyond the solution of specific problems to develop a more general study, which can permit a classification of the different possible types of trajectory. This is the new meaning of "solution" assigned to the qualitative treatment of differential equations. ${ }^{56}$ The field of dynamical systems emerges from this conception of what to "solve" a dynamical problem

\footnotetext{
55 Yoccoz (2007) shows that in all cases in which it is possible to understand something about the behavior of a set of trajectories, the description ultimately depends on hyperbolicity or quasi-periodicity of trajectories.

56 Chenciner (2007) suggests that the possibility of a global qualitative description of trajectories can be seen as a kind of integrability. This means that any system defining a (qualitatively) well-understood space of trajectories can be considered as fully integrable.
} 
means, leading to a separation from the traditional domains of differential equations and celestial mechanics.

Acknowledgments I am grateful to Frédéric Brechenmacher, José Ferreiròs, and Marco Panza for having read the first version of this article and made valuable suggestions. Any remaining problems are of my full responsibility. The author was partially supported with grants received from FAPERJ (Fundação de Amparo à Pesquisa do Estado do Rio de Janeiro) by means of the program Jovem Cientista do Nosso Estado.

Open Access This article is distributed under the terms of the Creative Commons Attribution Noncommercial License which permits any noncommercial use, distribution, and reproduction in any medium, provided the original author(s) and source are credited.

\section{References}

Anderson, K.G. 1994. Poincaré's discovery of homoclinic points. Archive for History of Exact Sciences 48(2):133-147.

Barrow-Green, J. 1994. Oscar II's prize competition and the error in Poincaré's memoir on the three body problem. Archive for History of Exact Sciences 48:107-131.

Barrow-Green, J. 1997. Poincaré and the Three Body Problem. Providence: American Mathematical Society, London Mathematical Society.

Barrow-Green, J. 2010. The dramatic episode of Sundman. Historia Mathematica 37:164-203.

Birkhoff, G.D. 1912. Quelques théorèmes sur le mouvement des systèmes dynamiques. Bulletin de la Société mathématique de France 40:305-323. In Birkhoff (1950), t. 1, 654-672.

Birkhoff, G.D. 1913. Proof of Poincaré's geometric theorem. Transactions of the American Mathematical Society 14:14-22. In Birkhoff (1950), t. 1, 673-681.

Birkhoff, G.D. 1915. The restricted problem of three bodies. Rendiconti del Circolo Matematico di Palermo 39:1-70.

Birkhoff, G.D. 1917. Dynamical systems with two degrees of freedom. Transactions of the American Mathematical Society 18:199-300. In Birkhoff (1950), t. 2, 1-102.

Birkhoff, G.D. 1920a. Recent advances in dynamics. Science (N.S.) 51(1307):51-55. In Birkhoff (1950), t. 2, 106-110.

Birkhoff, G.D. 1920b. Surface transformations and their dynamical applications. Acta Mathematica 43:1119. In Birkhoff (1950), t. 2, 111-129.

Birkhoff, G.D. 1925. An extension of Poincaré's last geometric theorem. Acta Mathematica 47:297-311. In Birkhoff (1950), t. 2, 252-266.

Birkhoff, G.D. 1926a. Sur la signification des équations canoniques de la dynamique. Comptes rendus de l'Académie des Sciences 183:516-519. In Birkhoff (1950), t. 2, 279-282.

Birkhoff, G.D. 1926b. Über gewisse zentralbewegungen dynamischer systeme. Ges. d. Wiss. Nachrichten. In Birkhoff (1950), t. 2, 283-294.

Birkhoff, G.D. 1927a. Dynamical Systems. Providence: American Mathematical Society. Reprinted in 1966 with additional references by Jurgen Moser.

Birkhoff, G.D. 1927b. Stability and the equations of dynamics. American Journal of Mathematics 49:1-38. In Birkhoff (1950), t. 2, 295-332.

Birkhoff, G.D. 1934. The work of Poincaré on differential equations. American Mathematical Society Bulletin 40:363-366. In Birkhoff (1950), t. 3, 544-547.

Birkhoff, G.D. 1935. Nouvelles recherches sur les systèmes dynamiques. Memoriae Pont. Acad. Sci. Novi Lyncaei 1(3):65-216. In Birkhoff (1950), t. 2, 530-661.

Birkhoff, G.D. 1950. Collected Mathematical Papers. Providence: American Mathematical Society. Reprinted in 1968, New York: Dover.

Brechenmacher, F. 2007. L'identité algébrique d'une pratique portée par la discussion sur l'équation à l'aide de laquelle on détermine les inégalités séculaires des planètes (1766-1874). Sciences et Techniques en Perspective 1:5-85.

Cesari, L. 1959. Asymptotic Behavior and Stability Problems in Ordinary Differential Equations. BerlinGöttingen: Springer. 
Chabert, J.-L., and A. Dahan-Dalmedico. 1992. Les idées nouvelles de Poincaré. In Dahan-Dalmedico et al. (1992), 274-305.

Chenciner, A. 2007. De la mécanique céleste à la théorie des systèmes dynamiques, aller et retour: Poincaré et la géométrisation de l'espace des phases. In Franceschelli et al. (2007), 13-36.

Dahan-Dalmedico, A. 1996. Le difficile héritage de Henri Poincaré en systèmes dynamiques. In Greffe et al. (1996), 13-33.

Dahan-Dalmedico, A., J.-L. Chabert, and K. Chemla, eds. 1992. Chaos et déterminisme. Paris: Seuil.

Darrigol, O. 2002. Stability and instability in nineteenth-century fluid mechanics. Revue d'histoire des mathématiques 8: 5-65.

Dell'Aglio, L. 1993. Tradizioni di ricerca nella meccanica celeste classica: il problema dei tre corpi in Levi-Civita e Sundman. Physis. Rivista Internazionale di Storia della Scienza 30(1):105-144.

Dell'Aglio, L., and G. Israel. 1987. I temi della stabilità e dell'analisi qualitativa nell'opera di Levi-civita et di Volterra. In La Matematica tra le Due guerre mondiali, ed. A. Guerraggio, 125-142. Bologna: Pitagora.

Dell'Aglio, L., and G. Israel. 1989. La théorie de la stabilité et l'analyse qualitative des équations différentielle ordinaires dans les mathématiques italliennes: le point de vue de Tullio Levi-Civita. Cahiers du séminaire d'histoire des mathématiques 10:283-321.

Franceschelli, S., M. Paty, and T. Roque, eds. 2007. Chaos et Systèmes Dynamiques. éléments pour une épistémologie. Paris: Hermann.

Gilain, C. 1977. La théorie géométrique des équations différentielles de Poincaré et l'histoire de l'analyse. Thèse de doctorat, Université de Paris7.

Gilain, C. 1991. La théorie qualitative de Poincaré et le problème de l'intégration des équations différentielles. In La France mathématique. La Société Mathématique de France (1872-1914), ed. H. Gispert, 215-242. Paris: Société Française d'Histoire des Sciences et des Techniques et Société Mathématique de France.

Grattan-Guinness, I. 1997. The Norton History of the Mathematical Sciences. New York: W.W. Norton \& Company.

Grattan-Guinness, I., and R. Cooke, eds. 2005. Landmark Writings in Western Mathematics (1640-1940). Amsterdam: Elsevier.

Gray, J. 1992. Poincaré, topological dynamics, and the stability of the solar system. In Harman and Shapiro (1992), 502-524.

Greffe, J.-L., G. Heinzmann, and K. Lorenz. eds. 1996. Henri Poincaré: Science et Philosophie. Paris: Blanchard, Akademie Verlag.

Gutzwiller, M.C. 1998. Moon-Earth-Sun: The oldest three-body problem. Reviews of Modern Physics 70(2):589-639.

Hadamard, J. 1901. Sur l'itération et les solutions asymptotiques des équations différentielles. Bulletin de la Société mathématique de France 29:224-228.

Harman, P.M., and A.E. Shapiro. 1992. The investigation of difficult things: Essays on Newton and the history of exact sciences in honour of D. T. Whiteside. Cambridge, UK: Cambridge University Press.

Hill, G.W. 1878. Researches in the lunar theory. American Journal of Mathematics 1(5):129-145.

La Salle, J., and S. Lefschetz. 1961. Stability of Lyapunov's Direct Method. New York: Academic Press.

Lagrange, J.L. 1774. Recherches sur les équations séculaires des mouvements des noeuds et des inclinaisons des orbites des planètes. Mémoires de l'Académie Royale des Sciences de Paris. In Lagrange (1867-1892), t. 6, 635-709.

Lagrange, J.L. 1781-1782. Théorie des variations séculaires des éléments des planètes. Nouveaux mémoires de l'Académie Royale des Sciences et Belles-Lettres de Berlin. In Lagrange (1867-1892), t. 5, 127-207, 208-344.

Lagrange, J.L. 1867-1892. Euvres de Lagrange. Paris: Gauthier-Villars.

Lagrange, J.L. 1788. Mécanique analytique. Paris: La Veuve Desaint. Reprinted in 1989. Paris: Jacques Gabay.

Laplace, P.-S. 1796. Exposition du système du monde. Paris: Impr. du Cercle-Social. In Laplace (18781912), t. 6.

Laplace, P.-S. 1878-1912. Euvres complètes de Laplace. Paris: Gauthier-Villars.

Laplace, P.-S. 1891-1904. Traité de Mécanique Céleste. Paris: Gauthier-Villars. In Laplace (1878-1912), t. $1-5$.

Laskar, J. 1992. La stabilité du système solaire. In Dahan-Dalmedico et al. (1992), 170-211. 
Lattès, S. 1906. Sur les équations fonctionnelles qui définissent une courbe ou une surface invariante par une transformation. Annali di Matematica Pura i Applicata 13: 1-138.

Le Verrier, U.-J. 1856. Annales de l'Observatoire de Paris, vol. 2. Paris: Mallet Bachelet.

Leine, R. 2010. The historical development of classical stability concepts: Lagrange, Poisson and Lyapunov stability. Nonlinear Dynamics 59: 173-182.

Lejeune-Dirichlet, J.P.G. 1846. Über die stabilität des gleichgewichts. Journal für die reine und angewandte Mathematik 32:85-88.

Levi-Civita, T. 1901. Sopra alcuni criteri di instabilità. Annali di Matematica Pura i Applicata 5:221-308.

Levi-Civita, T. 1920. Sur la régularisation du problème des trois corps. Acta Mathematica 42:99-144.

Lützen, J. 1984. Joseph Liouville's work on the figures of equilibrium of a rotating mass of fluid. Archive for History of Exact Sciences 30(2):113-166.

Lyapunov, A.M. 1897. Sur l'instabilité de l'équilibre dans certains cas où la fonction de forces n'est pas un maximum. Journal des Mathématiques Pures et Appliquées (5e série) III:81-94.

Lyapunov, A.M. 1904. Sur la stabilité des figures ellipsoïdales d'équilibre d'un liquide animé d'un mouvement de rotation. Annales de la Faculté des Sciences de l'Université de Toulouse (2) 6:5-116.

Lyapunov, A.M. 1907. Problème général de la stabilité du mouvement (translated by E. Davaux). Annales de la Faculté des Sciences de l'Université de Toulouse (2) 9: 203-474. Reprinted in 1988, Paris: Jacques Gabay.

Lyapunov, A.M. 1992. The General Problem of the Stability of Motion. Washington: Taylor \& Francis.

Mawhin, J. 1994. The centennial legacy of Poincaré and Lyapunov in ordinary differential equations. Rendiconti del Circolo Matematico di Palermo (II) 34:9-46.

Mawhin, J. 1996. The early reception in france of the work of Poincaré and Lyapunov in the qualitative theory of differential equations. Philosophia Scientia 1(4):119-133.

Mawhin, J. 2005. Aleksandr Mikhailovich Lyapunov thesis on the stability of motion (1892). In GrattanGuinness and Cooke (2005), (eds.) 664-676.

Morse, M. 1925. Relations between the critical points of a function of $n$ independent variables. Transactions of the AMS 27:345-396.

Nabonnand, P. 2000. Les recherches sur l'œuvre de Poincaré. SMF-Gazette 85:33-54.

Nastasi, P., and R. Tazzioli. eds. 2000. Aspetti scientifici e umani nella corrispondenza di Tullio Levi-Civita (1873-1941), n. 12. Palermo: Quaderno PRISTEM.

Poincaré, H., and G. Mittag-Leffler. 1999. La correspondance entre Henri Poincaré and Gösta MittagLeffler, avec en annexe les lettres échangées par Poincaré avec Fredholm, Gyldèn et Phragmén. Basel: Birkhaüser. Published by P. Nabonnand.

Poincaré, H. 1881. Mémoire sur les courbes définies par une équation différentielle (1 ${ }^{\text {re }}$ partie). Journal de Mathématiques (3e série) 7:375-422. In Poincaré (1951-1956), t. I, 3-44.

Poincaré, H. 1882. Mémoire sur les courbes définies par une équation différentielle ( $2^{e}$ partie). Journal de Mathématiques ( $3^{e}$ série) 8:251-296. In Poincaré (1951-1956), t. I, 44-84.

Poincaré, H. 1885a. Mémoire sur les courbes définies par une équation différentielle ( $3^{e}$ partie). Journal de Mathématiques (4e série) 1:167-244. In Poincaré (1951-1956), t. I, 90-158.

Poincaré, H. 1885b. Sur l'équilibre d'une masse fluide animée d'un mouvement de rotation. Acta Mathematica 7:159-380. In Poincaré (1951-1956), t. 7, 40-140.

Poincaré, H. 1886. Mémoire sur les courbes définies par une équation différentielle (4 ${ }^{e}$ partie). Journal de Mathématiques (4e série) 2:151-217. In Poincaré (1951-1956), t. I, 167-222.

Poincaré, H. 1889. Sur le problème des trois corps et les équations de la dynamique avec des notes par l'auteur-Mémoire couronné du prix de S.M. le Roi Oscar II. Not published.

Poincaré, H. 1890. Sur le problème des trois corps et les équations de la dynamique. Acta Mathematica 13:1-270. In Poincaré (1951-1956), t. 7, 262-479.

Poincaré, H. 1892-1899. Méthodes nouvelles de la mécanique céleste. Paris: Gauthier-Villars.

Poincaré, H. 1912. Sur un théorème de géométrie. Rendiconti dei circolo matematico di Palermo 33:375407. In Poincaré (1951-1956), t. 6, 499-538.

Poincaré, H. 1951-1956. Euvres d'Henri Poincaré. Ed. Paul Appel et al. Paris: Gauthier-Villars.

Poisson, S.D. 1808. Mémoire sur les inégalités séculaires des moyens mouvements des planètes. Journal de l'école Polytechnique 15(1): 1-56.

Robadey, A. 2006. Différentes modalités de travail sur le général dans les recherches de Poincaré sur les systèmes dynamiques. Thèse de doctorat, Université Paris7.

Roque, T. 2007. Les enjeux du qualitatif dans la définition d'un système dynamique. In Franceschelli et al. (2007), 37-66. 
Smirnov, V.I., and A.P. Youchkevitch. 1987. Correspondence de A. M. Liapunov avec H. Poincaré. Cahiers du Séminaire d'Histoire des Mathématiques 8:1-18. www.numdam.org.

Sundman, K.F. 1907. Recherches sur le problème des trois corps. Acta Societatis Scientiarum Fennicae 34:1-43.

Sundman, K.F. 1909. Nouvelles recherches sur le problème des trois corps. Acta Societatis Scientiarum Fennicae 35:1-27.

Sundman, K.F. 1912. Mémoire sur le problème des trois corps. Acta Mathematica 36:105-179.

Taton, R., C. Wilson, and M. Hoskin. 2009. The general history of astronomy, vol. 2: Planetary astronomy from the renaissance to the rise of astrophysics. Cambridge: Cambridge University Press.

Thomson, W., and P.G. Tait. 1879-1883. Treatise on Natural Philosophy (2nd ed). Cambridge: Cambridge University Press.

Tisserand, F. 1889-1896. Traité de Mécanique Céleste. Paris: Gauthier-Villars et fils. Reprinted in 1990, Paris: Jacques Gabay.

Whittaker, E.T. 1917. A treatise on the analytical dynamics of particles and rigid bodies, 2nd edn. Cambridge: Cambridge University Press.

Wise, N. 2005. William Thomson and Peter Guthrie Tait, Treatise on natural philosophy, 1st edn (1867). In Grattan-Guinness and Cooke (2005), 521-533.

Yoccoz, J.C. 2007. Hyperbolicité et quasipériodicité. In Franceschelli et al. (2007), 145-156. 